MPIfG Journal Article

Guido Möllering: Umweltbeeinflussung durch Events? Institutionalisierungsarbeit und feldkonfigurierende Veranstaltungen in organisationalen Feldern. In: Schmalenbachs Zeitschrift für Betriebswirtschaftliche Forschung (63), 458 - 484 (2011). Verlagsgruppe Handelsblat

Guido Möllering*

The MPIfG Journal Articles series features articles by MPIfG researchers and visiting scholars published in peer-reviewed journals. Max Planck Institute for the Study of Societies (MPIfG) Cologne | www.mpifg.de

\title{
Umweltbeeinflussung durch Events? Institutionalisierungsarbeit und feldkonfigurierende Veranstaltungen in organisationalen Feldern ${ }^{* *}$
}

\section{Zusammenfassung}

Der Beitrag untersucht zwei neue Konzepte in der Neoinstitutionalistischen Organisationstheorie: Institutionalisierungsarbeit (Institutional Work) und feldkonfigurierende Veranstaltungen (Field-Configuring Events). Diese Theorieströmung vollzieht damit eine praxistheoretische Wende und gesteht Unternehmen mehr Möglichkeiten der Umweltbeeinflussung zu als der bisherige neoinstitutionalistische Determinismus, ohne jedoch in den methodologischen Individualismus zu verfallen. Der Beitrag untersucht kritisch die Quellen und Hintergründe dieser Theorieentwicklung und kommt zu dem vorsichtig optimistischen Fazit, dass es für die Betriebswirtschaftslehre ein theoretischer und methodischer Gewinn ist, Management als Institutionalisierungsarbeit zu verstehen.

JEL-Classification: D02, M19, Z13.

Keywords: Event; Field; Institution; Neoinstitutional Theory; Organization.

Feld; Institution; Neoinstitutionalistische Theorie; Organisation; Veranstaltung.

\section{Einleitung}

Neueste Entwicklungen in der Neoinstitutionalistischen Organisationstheorie werfen wieder einmal die Frage auf, inwieweit Unternehmen sich Umweltbedingungen anpassen müssen oder umgekehrt diese Bedingungen beeinflussen können. Dies ist nach wie vor ein wichtiges Thema für eine Betriebswirtschaftslehre, die sich als anwendungsorientierte Wissenschaft versteht und den Anspruch erhebt, gestaltungsrelevante Aussagen zu treffen $^{1}$. Es gilt zu ermessen, wie weit die Gestaltungsspielräume von Entscheidungs-

* Prof. Dr. Guido Möllering, Professor für Organisation und Management, Jacobs University Bremen, Campus Ring 1, 28759 Bremen, Tel.: 0421/200-3035, Fax: 0421/200-3303, E-Mail: g.moellering@jacobs-university.de.

** Der Autor dankt Prof. Dr. Jörg Sydow, Prof. Dr. Peter Walgenbach, denen, die beim Habilitationsvortrag am Fachbereich Wirtschaftswissenschaft der Freien Universität Berlin mitdiskutiert haben, und dem anonymen Gutachter der $z f b f$ für wertvolle Hinweise und Anregungen. Die Arbeit an dem Artikel wurde unterstützt durch das Max-Planck-Institut für Gesellschaftsforschung und durch Daten aus einem Projekt der VolkswagenStiftung (AZ II/80 308).

1 Vgl. Wöhe/Döring (2010), S. 27. 
trägern in Unternehmen reichen und wo deren Grenzen liegen. Dieser Beitrag untersucht zwei neuere Konzepte, bei denen es darum geht, inwieweit Wirtschaftssubjekte die Grenzen ihrer Spielräume gestalten können: Institutionalisierungsarbeit und feldkonfigurierende Veranstaltungen. Das Konzept der Institutionalisierungsarbeit (Institutional Work $)^{2}$ verweist auf die Beeinflussbarkeit von Regelsystemen in der institutionellen Umwelt von Unternehmen. Und das Konzept der feldkonfigurierenden Veranstaltungen (Field-Configuring Events) $^{3}$ meint Veranstaltungen, die ein organisationales Feld prägen und bei denen Unternehmen Institutionalisierungsarbeit leisten können.

Beide Konzepte werden im deutschsprachigen Raum bisher allenfalls unter einigen Spezialisten diskutiert, verdienen aber eine breitere Auseinandersetzung innerhalb der Betriebswirtschaftslehre. Neoinstitutionalistische Theorieansätze in ihrer Gesamtheit gehören seit einiger Zeit zu den dominanten Strömungen unter den Organisationstheorien ${ }^{4}$. Bei den Academy of Management Meetings liegt keine andere Theorie mehr Beiträgen im Bereich Organisations- und Management Theorie (OMT) zugrunde als der Neoinstitutionalismus ${ }^{5}$. Die Diskussionen um Institutionalisierungsarbeit und feldkonfigurierende Veranstaltungen sind somit von zentraler Bedeutung für die heutige Betriebswirtschaftslehre. Dies wird nur bestätigt durch die Tatsache, dass die Probleme, mit denen sich Neoinstitutionalisten aktuell beschäftigen, im Grunde genommen klassische sind, die aber keineswegs zuvor schon abschließend gelöst wurden ${ }^{6}$.

Was genau dahinter steckt, wird im Folgenden analysiert. Zuvor mag ein kurzes empirisches Beispiel, auf das ich in diesem Beitrag immer wieder zurückkommen werde, der Illustration dienen: In der Halbleiterindustrie war es seit Jahrzehnten selbstverständlich, dass Chips nach dem Verfahren der sogenannten Optischen Lithographie hergestellt werden $^{7}$. Da dieses Paradigma jedoch an physikalische Grenzen stößt, arbeiten die ChipHersteller und ihre Lieferanten an Alternativen und das heißt, sie arbeiten nicht nur im engeren Sinn an neuer Technologie, sondern auch an der neuen Architektur und den künftigen Spielregeln des unternehmensübergreifenden Produktionszusammenhangs: Das ist zusammen genommen als Institutionalisierungsarbeit zu bezeichnen, die in diesem Feld zu einem großen Teil weltweit über Konsortien geleistet wird. In diesem Prozess ist auffällig, dass regelmäßig Kongresse veranstaltet werden, an denen alle relevanten Unternehmen teilnehmen und bei denen versucht wird, Klarheit über die zukünftige Technologie und deren Konsequenzen für Märkte, Supply Chains und Unternehmen zu schaffen ${ }^{8}$. Diese Kongresse sind, zumindest potenziell, feldkonfigurierende Veranstaltungen und geben Unternehmen Gelegenheit zur strategischen Beeinflussung des Feldes.

2 Vgl. Lawrence/Suddaby (2006).

3 Vgl. Lampel/Meyer (2008).

4 Greenwood et al. (2008), S. 2: „Institutional theory is perhaps the dominant approach to understanding organizations."Vgl. auch Walgenbach (2006), S. 389.

5 Dies ist zumindest für das Jahr 2005 durch Davis (2006), S. 114f. belegt und wird bei den AOM-Jahrestagungen und anderen Gelegenheiten (zum Beispiel Podiumsdiskussionen zum Neoinstitutionalismus) immer wieder informell bestätigt, vgl. auch Lawrence/Suddaby/Leca (2011), S. 52.

6 Vgl. Lawrence/Suddaby/Leca (2011), S. 53: „These questions are not, of course, unique to the concept of institutional work."

7 Vgl. Möllering (2010), zur Halbleiterindustrie im Allgemeinen vgl. Brown/Linden (2009).

8 Vgl. Dao/Mackay/Seidel (2002). 
Ziel des Beitrags ist, den Erkenntnisbeitrag der Literatur zu Institutional Work und FieldConfiguring Events kritisch auszuloten. Im Vordergrund steht die Frage, inwieweit die Neoinstitutionalistische Organisationstheorie, der die beiden Konzepte zuzurechnen sind', sich weiterentwickelt und ob ihre Relevanz dadurch für die betriebswirtschaftliche Forschung und Praxis größer wird. Ich erläutere nun den Begriff des organisationalen Feldes und damit die zentralen Grundlagen und Bezugspunkte meiner Analyse in der Neoinstitutionalistischen Organisationstheorie ${ }^{10}$. Dann widme ich mich zuerst der Literatur zur Institutionalisierungsarbeit und danach den feldkonfigurierenden Veranstaltungen, bevor ich zu einer kritischen Schlussbetrachtung komme.

\section{Organisationale Felder}

\subsection{Zum Begriff des organisationalen Feldes}

Jede Organisation, also auch jedes Unternehmen, ist in der Sichtweise des Neoinstitutionalismus Teil mindestens eines sogenannten organisationalen Feldes ${ }^{11}$. Diesen Begriff definieren DiMaggio und Powell als diejenigen Organisationen, die in ihrer Gesamtheit einen anerkannten Bereich institutionellen Lebens konstituieren: wichtige Lieferanten, Konsumenten, Regulatoren und andere Organisationen, die ähnliche Güter produzieren (eigene Übersetzung) ${ }^{12}$. Das bedeutet also erstens: Organisationale Felder bestehen aus Organisationen; jede Organisation ist Teil ihres Feldes; und Organisationen setzen sich somit nicht von außen, sondern von innen mit ihrem Feld auseinander. Zweitens besteht ein organisationales Feld in deutlicher Erweiterung etwa des Begriffes der Industrie oder der Branche nicht nur aus den Organisationen, die ähnliches produzieren, sondern auch aus deren Lieferanten und Kunden sowie aus staatlichen und anderen Einrichtungen, die das Feld regulieren können. Drittens ist in der Definition der Verweis auf „a recognized area of institutional life" wichtig, der jedoch in dieser Formulierung schwammig bleibt.

Dieser Aspekt wird in der zweiten gängigen Definition des Begriffes von Scott um einiges deutlicher. Scott schreibt: „Mit dem Begriff des Feldes ist verbunden, dass eine Gemeinschaft von Organisationen existiert, die an einem gemeinsamen Sinnsystem beteiligt ist und deren Teilnehmer öfter und folgenreicher miteinander interagieren als mit Akteuren außerhalb des Feldes“ (eigene Übersetzung) ${ }^{13}$. Mit dieser Definition kann zum einen jede

9 Bei Institutional Work ist diese Zurechnung klar. Bei den Field-Configuring Events wäre auch eine Zuordnung zu anderen Feldtheorien denkbar, jedoch verweisen Lampel/Meyer (2008), S. 1027 eindeutig auf einen neoinstitutionalistischen Theoriehintergrund.

10 Der Begriff des organisationalen Feldes gilt als zentral für die Neoninstitutionalistische Organisationstheorie, vgl. u.a. Scott (2008), S. 181, Wooten/Hoffman (2008), S. 130.

11 Vgl. zum Thema „organisationale Felder“ neben der in diesem Abschnitt rezipierten Literatur auch Walgenbach (2006), S. 368f.; Wooten/Hoffman (2008).

12 DiMaggio/Powell (1983), S. 143: „By organizational field we mean those organizations that, in the aggregate constitute a recognized area of institutional life: key suppliers, resource and product consumers, regulatory agencies, and other organizations that produce similar services or products."

13 Scott (1994), S. 207f.: "The notion of field connotes the existence of a community of organizations that partakes of a common meaning system and whose participants interact more frequently and fatefully with one another than with actors outside the field." 
Art von Organisation einem Feld angehören, also etwa auch eine Umweltschutzorganisation dem Feld der Energiegewinnung. Denn zum anderen geht es konzeptuell immer weniger darum, von außen harte, gar materielle Kriterien für die Feldzugehörigkeit vorzugeben - zum Beispiel welches Produkt oder Leistungsbündel ein Unternehmen herstellt und verkauft ${ }^{14}$. Stattdessen wird auf ein Sinnsystem verwiesen (siehe Abbildung 1). Mit „Sinnsystem“ sind formale und informelle Regeln, Wertvorstellungen, Annahmen über die Welt und alle Formen von Wissen gemeint, die für die Organisationen in einem Feld von Bedeutung sind. Typisch für das Sinnsystem der Halbleiterindustrie zum Beispiel sind der hohe Innovationsdruck gemäß „Moore's Law“" ${ }^{15}$, die optische Lithographie als technologisches Paradigma oder die zunächst sehr strenge, dann aber gelockerte Regulation von Kartellen und Unternehmenskooperationen.

\section{Abbildung 1: Organisationales Feld nach Scott (Schematische Darstellung)}

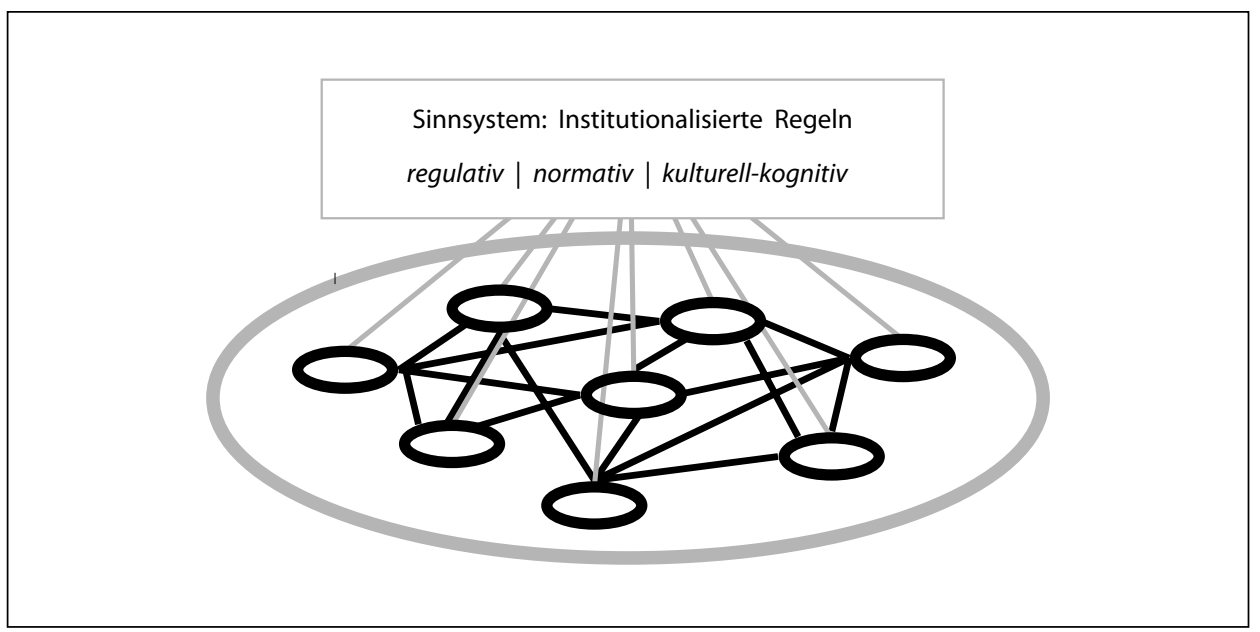

Im Verständnis dieser Theorie hängt die Zugehörigkeit zu einen organisationalen Feld also davon ab, ob eine Organisation sich an dem jeweiligen Sinnsystem orientiert. Organisationen, die das gleiche Sinnsystem anwenden, interagieren häufiger miteinander. Durch diese Interaktion verfestigen sie wiederum das Sinnsystem sowie die Abgrenzung des Feldes von anderen und werden so als "Gemeinschaft "16 interagierender Organisationen erkennbar ${ }^{17}$. Einige Aspekte des hier vorgestellten Feldbegriffes finden sich auch in anderen Theorien, doch die Neoinstitutionalisten betonen die Ebene des Sinnsystems, welches als System institutionalisierter Regeln zu verstehen ist. Die industrieökonomische

14 Hoffman (1999) zeigt zum Beispiel, dass organisationale Felder auch um strittige Themen (,issues“) entstehen können.

15 Hiermit ist eine Produktivitätssteigerungsregel gemeint, die Moore (1965) aufstellte, wonach sich die Leistung von Computerchips etwa alle 18 Monate verdoppeln werde (oder sollte).

16 Scott (1994), S. 207.

17 So müsste man eigentlich treffender von interorganisationalen Feldern sprechen, Warren (1967). 
Abgrenzung anhand von Produkten ${ }^{18}$ könnte dennoch angemessen sein, weil sich in vielen Feldern das Sinnsystem stark, allerdings nicht ausschließlich, auf das Produkt bezieht. Im Neoinstitutionalismus werden die Grenzen nicht auf der materiellen Ebene der Konkurrenz um Markttransaktionen gezogen, sondern auf der Ebene übergeordneter Regeln, auf die sich Unternehmen beziehen.

\subsection{Das Drei-Säulen-Modell von Scott}

Institutionalisierte Regelsysteme lassen sich nach Scott in drei Säulen gliedern (siehe Abbildung 1$)^{19}$. Die regulative Säule bezieht sich auf Gesetze, Verordnungen und andere „harte“ Regeln, deren Beachtung kontrolliert und sanktioniert wird und die von Unternehmen nutzenorientiert befolgt werden, um Strafen zu vermeiden oder Belohnungen zu erhalten. Beispiele in der Halbleiterindustrie wären das Wettbewerbsrecht, Patentrecht oder auch Forschungsförderprogramme. In der Nahrungsmittelbranche ist zum Beispiel gesetzlich reguliert, wieviel Dioxin ein Produkt enthalten darf, und wer sich nicht daran hält, handelt illegal - wir erinnern uns an den Skandal im Januar 2011. Hier liegt Scotts Modell noch größtenteils auf einer Linie mit der Neuen Institutionenökonomie ${ }^{20}$. Anreizsysteme spielen also auch im Konzept der organisationalen Felder eine Rolle. Neoinstitutionalisten würden allerdings sagen, dass regulative Sanktionen nur dann als institutionalisiert gelten, wenn sie über ihre Nutzeneffekte hinaus akzeptiert und für selbstverständlich gehalten werden $^{21}$. Dies verweist auf die beiden anderen Säulen in Scotts Modell.

Die normative Säule umfasst Regeln, die aus gesellschaftlichen Werten abgeleitet sind und den Unternehmen zum Beispiel vorgeben, mit welchen Mitteln sie ihre Ziele zu erreichen versuchen dürfen. Die Säule trägt, wenn die Betroffenen sich zur Einhaltung der Regel verpflichtet fühlen, oder zumindest den Eindruck vermitteln ${ }^{22}$, ohne dass damit zwingend ihr Nutzen maximiert wird ${ }^{23}$. Wenn Unternehmen also kein Dioxin im Futter verarbeiten, so ist der Grund nicht allein, dass sie Geldstrafen und Insolvenz fürchten, sondern vielleicht auch, dass die Vergiftung von Endkonsumenten nicht den Wertvorstellungen entsprechen würde, denen sie sich moralisch verpflichtet fühlen. Sie befolgen die normative Regel, dass Gewinne nicht durch die Verwendung von Gift maximiert werden dürfen. Auch in der Halbleiterindustrie wirken normative Regeln wie zum Beispiel Regeln der Vertraulichkeit unter Kooperationspartnern, die juristisch schwer durchsetzbar, aber in gewisser Weise „Ehrensache“ sind.

18 Vgl. die International Standard Industrial Classification (ISIC) der Vereinten Nationen.

19 Vgl. Scott (2008), S. 47ff., außerdem die Darstellung bei Walgenbach (2006), S. 378ff. Die Säulen zusammen tragen die Institutionen, jedoch ruht nicht jede Institution in gleichem Maße auf allen drei Säulen. Vielmehr lassen sich mit Scotts Drei-Säulen-Modell Idealtypen von Institutionen unterscheiden. In der Realität weisen institutionalisierte Regeln dann die Merkmale der Säulen unterschiedlich stark auf. Zur Kritik am Drei-SäulenModell vgl. u.a. Walgenbach (2002).

20 Zum Verhältnis von Neoinstitutionalismus und Neuer Institutionenökonomie, insbes. deren vordergründigen Gemeinsamkeiten, aber auch fundamentalen Unterschieden, vgl. Walgenbach/Meyer (2008b), S. 151ff.

21 Vgl. dazu bereits Zucker (1977; 1987).

22 Auf die von Meyer/Rowan (1977) prominent entwickelten Konzepte der Entkopplung und der vorwiegend symbolischen Regelkonformität als Fassade kann hier nicht weiter eingegangen werden.

23 Vgl. Walgenbach (2006), S. 380. 
Die dritte Säule bezeichnet Scott als kulturell-kognitive Säule. Diese Säule trifft am deutlichsten den Aspekt des gemeinsamen Sinnsystems in einem organisationalen Feld. Kulturell-kognitive Institutionen regeln nämlich, wie Akteure die Wirklichkeit sinnhaft erschließen. Bestimmte denkbare Verhaltensweisen ergeben somit keinen Sinn und kommen den Akteuren gar nicht in den Sinn ${ }^{24}$. Diese Institutionen sind vielleicht am besten als Rollen, Routinen oder Skripte vorstellbar, die unhinterfragt ausgeführt werden $^{25}$. In der Halbleiterindustrie gibt es viele solcher kulturell-kognitiver Regeln, die aus der natur- und ingenieurwissenschaftlichen Sozialisation der Entscheider stammen. Sie sehen alles zuerst durch die Brille der Physik und in der Halbleiterindustrie insbesondere durch die Brille der optischen Physik, in der sich alle Probleme und deren Lösungen um Licht drehen. Und wegen solcher kognitiver Regeln mag auch, nun in einem ganz anderen Feld, ein Nahrungsmittelunternehmer routinemäßig kein Dioxin verwenden, weil das für ihn unvorstellbar wäre, und nicht, weil er Bestrafung, Ächtung oder ein schlechtes Gewissen fürchtet. Dioxin passt nicht zu seiner Rolle als Nahrungslieferant. Ein organisationales Feld zeichnet sich dadurch aus, dass die Organisationen in ihm die gleichen oder zumindest sehr ähnliche - Rollenverständnisse und -erwartungen haben.

\subsection{Jenseits des institutionellen Isomorphismus}

Lange Zeit war die wohl wichtigste Hypothese der Neoinstitutionalisten ${ }^{26}$, dass sich die Organisationen innerhalb eines Feldes mit der Zeit immer ähnlicher werden, gerade weil sie sich auf ein gemeinsames Sinnsystem beziehen und den gleichen Regeln gerecht werden müssen, um Legitimität zu erlangen ${ }^{27}$. Jede von Scotts Säulen geht mit einem der von DiMaggio und Powell benannten Mechanismen der institutionellen Isomorphie einher ${ }^{28}$. Dieser Isomorphismus ist es, der den Neoinstitutionalisten den Ruf einbrachte, sie propagierten einen Determinismus, der keinen Raum für echte unternehmerische Entscheidungen lässt und die spezifischen Voraussetzungen und ökonomisch-technischen Effizienzerfordernisse einzelner Unternehmen trivialisier ${ }^{29}$. Doch auch wenn man akzeptiert, dass Institutionen stark beeinflussen, wie Unternehmen handeln, gibt es noch viele offene Fragen, mit denen sich die Neoinstitutionalisten selbst seit mindestens zwanzig Jahren plagen ${ }^{30}$ und an deren Beantwortung sich die Betriebswirtschaftslehre konstruktiv beteiligen kann: Wie entstehen Institutionen? Wirken Institutionen auf alle Organisationen in einem Feld gleich? Oder: Reagieren alle Organisationen in gleicher Weise auf gegebene Institutionen? Wie kommt es zu institutionellem Wandel? Und vor allem:

24 Vgl. neben der Darstellung bei Scott (2008), S. 56ff., bereits Zucker (1977).

25 Vgl. Berger/Luckmann (1966).

26 Vgl. Wooten/Hoffman (2008), S. 134.

27 Vgl. Meyer/Rowan (1977); DiMaggio/Powell (1983).

28 Vgl. Scott (2008), S. 50ff. und dort in Tabelle 3.1 die Mechanismen von DiMaggio/Powell (1983); vgl. auch Walgenbach (2006), S. 368ff. und 378ff.

29 Walgenbachs (2006) Darstellung der neoinstitutionalistischen Ansätze lässt diesen Ruf als durchaus gerechtfertigt erscheinen. Auch in einigen der neuesten programmatisch angelegten Aufsätze zur Neoinstitutionalistischen Organisationstheorie wird die nachdrückliche Behauptung der „primacy of institutions“ gefordert, vgl. Suddaby et al. (2010), S. 1236.

30 Vgl. zu den hier relevanten, neueren Themen der neoinstitutionalistischen Literatur zum Beispiel Greenwood et al. (2008); Suddaby (2010); Walgenbach (2002; 2006), S. 390ff.; Wooten/Hoffman (2008). 
Können Organisationen Institutionen beeinflussen? Um diese Fragen geht es auch in der Literatur zu Institutionalisierungsarbeit (Institutional Work).

\section{Institutionalisierungsarbeit}

\subsection{Vorbemerkungen zur Institutional Work-Literatur}

Neu ist Institutionalisierungsarbeit vor allem in der Hinsicht, dass es seit 2006 eine Reihe von Veröffentlichungen gibt, die den Begriff Institutional Work prominent verwenden und ausarbeiten. Nicht ohne eine gewisse Ironie arbeiten die beteiligten Autoren daran, das Konzept der Institutionalisierungsarbeit zu institutionalisieren, und geben damit gleich ein gutes Beispiel für das, was sie konzeptualisieren ${ }^{31}$. Der Ausdruck „institutional work" taucht in einem sehr einflussreichen Aufsatz von DiMaggio bereits 1988 mehrfach auf und bezeichnet schon dort die Bemühungen von Akteuren, mit denen Institutionen aktiv reproduziert und erhalten werden oder aber auch neu erschaffen werden sollen ${ }^{32}$. Außerdem beansprucht die Literatur unter dem Label Institutional Work keinen gänzlich neuen Ansatz, sondern verspricht in erster Linie, einen Weg gefunden zu haben, verschiedene Aspekte aus den Debatten um institutionellen Wandel und Institutional Entrepreneurship sinnvoll zusammenbringen und weiterentwickeln zu können ${ }^{33}$. Dennoch haben wir es, wie ich im Folgenden zeigen werde, mit einem ernstzunehmenden Versuch zu tun, die Neoinstitutionalistische Organisationstheorie systematisch neu zu orientieren ${ }^{34}$.

\subsection{Zum Begriff der Institutionalisierungsarbeit}

Hauptreferenzpunkt für die aktuelle Diskussion über Institutionalisierungsarbeit ist ein 2006 im Sage Handbook of Organization Studies erschienener Aufsatz von Lawrence und Suddaby mit dem Titel „Institutions and Institutional Work“"35 und daneben ein

31 Dass diese Ironie den Vertretern selbst wohl nicht ganz verborgen bleibt, zeigt das Kapitel von Boxenbaum/ Strandgaard Pedersen (2009) über "Scandinavian institutionalism - a case of institutional work“ in einem Sammelband über Institutional Work.

32 Vgl. DiMaggio (1988), S. 13-15. Dieser Hinweis - vgl. auch Möllering (2010), S. 6 - fehlt allerdings bezeichnender Weise in den konzeptionellen Kernbeiträgen zu Institutional Work von Lawrence/Suddaby (2006) und Lawrence/Suddaby/Leca (2009); Kraatz (2011) meint gar, der Begriff sei neu.

33 Vgl. Lawrence/Suddaby (2006), S. 216: „Thus, a key contribution of this chapter will be the provision of a framework that connects previously disparate studies of institutional work and the articulation of a research agenda for the area." Lawrence/Suddaby/Leca (2009), S. 1: „Institutional work represents an exciting direction for institutional studies of organization, not because it represents a "new" idea, but because it connects a set of previously disparate ideas."

34 Vgl. Lawrence/Suddaby (2006), S. 246 und 249: „We have begun to document a new direction in institutional research - the study of institutional work ... a new way of seeing institutions. "Lawrence/Suddaby/Leca (2011), S. 52: „... an alternative to the path along which institutional theory has been developing. “ Phillips (2011), S. 51: „Institutional work ... has rapidly gained prominence. The number of studies of various kinds that rapidly appeared under the banner of institutional work ... all attest to its perceived usefulness and originality; " Hwang/ Colyvas (2011), S. 62: „Institutional work represents a significant reorientation of institutional theory.“

35 Vgl. Lawrence/Suddaby (2006). Diese Autoren wiederum nennen DiMaggio (1988) und Oliver (1991; 1992), als maßgebliche Quellen für ihren Ansatz. In einem früheren Aufsatz spricht Lawrence (1999) bereits von ,institutional strategy" als Handlungsmuster der Beeinflussung von Institutionen. 
Herausgeberband von 2009 bei Cambridge University Press ${ }^{36}$. In dem Handbuchbeitrag umschreiben die Autoren Institutionalisierungsarbeit als das absichtsvolle Handeln von Individuen und Organisationen, welches auf das Erschaffen, Erhalten und Erschüttern von Institutionen gerichtet ist (eigene Übersetzung) ${ }^{37}$. Aus dieser Definition geht bereits ein erster Anspruch hervor, den die Autoren erheben: Unter dem Begriff Institutionalisierungsarbeit sollen bisherige Forschungen zusammengebracht werden, die sich jeweils mit der Erschaffung von neuen Institutionen, mit der Reproduktion von bestehenden Institutionen oder mit Deinstitutionalisierungsprozessen befasst haben ${ }^{38}$.

Zweitens impliziert diese Definition die entscheidende Annahme, dass Individuen und Organisationen in jeder Phase der Institutionalisierung aktiv, durch ihr absichtsvolles Handeln, Einfluss auf Institutionen nehmen können oder dies zumindest versuchen ${ }^{39}$. Dies weicht in mehrerlei Hinsicht von gängigen Vorstellungen im Neoinstitutionalismus der stärker deterministischen Sorte ab:

(1) Bisher nahm man häufig an, Akteure könnten Institutionen nur so lange beeinflussen, wie sie noch im Entstehen begriffen sind. Haben sie sich hingegen erst einmal verfestigt, stehen Akteure ihnen nur noch passiv gegenüber ${ }^{40}$. Lawrence und Suddaby weisen hingegen darauf hin, dass Institutionalisierungsprozesse niemals völlig abgeschlossen sind und somit auch immer Beeinflussungsmöglichkeiten bestehen ${ }^{41}$.

(2) Außerdem betont diese Definition, dass das absichtsvolle Handeln der Akteure sich nicht zwingend gegen Institutionen richten muss, wie man den Eindruck haben könnte, wenn man den institutionellen Determinismus gegen die individuelle Handlungsfreiheit positioniert. Diesen Gegensatz stark zurücknehmend, sieht der Begriff der Institutionalisierungsarbeit auch vor, dass Akteure Institutionen, die für sie günstig sind, bewusst pflegen und erhalten ${ }^{42}$. Damit ist nicht einfach gemeint, dass sie bestehende Regeln befolgen und sie damit bestätigen, sondern es geht um Aktivitäten, die sicherstellen sollen, dass auch andere die Regeln befolgen und dass die Regeln nicht geändert werden ${ }^{43}$.

(3) Außerdem verabschieden sich Lawrence und Suddaby von der Vorstellung, institutioneller Wandel und das Verschwinden von Institutionen sowie jegliche Art von institu-

36 Vgl. Lawrence/Suddaby/Leca (2009). Eine weitere Darstellung und Pointierung des Konzeptes findet sich bei Lawrence/Suddaby/Leca (2011).

37 Lawrence/Suddaby (2006), S. 215: „... what we refer to as 'institutional work' - the purposive action of individuals and organizations aimed at creating, maintaining and disrupting institutions."

38 Vgl. Lawrence/Suddaby (2006), S. 216 und 218, die behaupten, es sei ,an important tradition within institutional theory that explores theoretically and empirically the ways in which actors are able to create, maintain and disrupt institutions" entstanden.

39 Vgl. Lawrence/Suddaby/Leca (2009), S. 1: „...shifting the focus to understanding how action affects institutions."

40 Nach Berger/Luckmann (1966) sind unhinterfragbare Selbstverständlichkeiten ja das Ergebnis von Institutionalisierungsprozessen; vgl. auch Walgenbach (2006), S. $354 \mathrm{ff}$.

41 Vgl. Lawrence/Winn/Jennings (2001); Lawrence/Suddaby (2006), S. 217.

42 Zur Frage der Intentionalität von Institutionalisierungsarbeit vgl. Lawrence/Suddaby/Leca (2009), S. 13f.

43 Vgl. Lawrence/Suddaby (2006), S. 229: „Relatively few institutions have such powerful reproductive mechanisms that no ongoing maintenance is necessary.“ 
tioneller Störung würden nur durch externe Schocks hervorgerufen. Stattdessen kann Institutionalisierungsarbeit auch von Innen betrieben werden ${ }^{44}$. So reifte etwa unter den Unternehmen in der Halbleiterindustrie selbst die Erkenntnis, dass eine technologische Sackgasse droht und man dementsprechend Initiativen für einen koordinierten Paradigmenwechsel ergreifen sollte.

Die Neoinstitutionalisten kommen mit dieser Konzeptualisierung der Betriebswirtschaftslehre ein großes Stück entgegen. Institutionalisierte Regeln sind für Unternehmen kein rigides Korsett mehr, kein stahlhartes Gehäuse auf der Makroebene, wie DiMaggio und Powell es in Anspielung auf Max Weber skizzierten ${ }^{45}$, sondern etwas, womit man sich auseinandersetzen kann. Die Frage ist nun weniger: Wie handelt ein Unternehmen optimal in einem gegebenen institutionellen Kontext? Stattdessen fragt man: Wie wirken die Aktivitäten eines Unternehmens auf den institutionellen Kontext und wird er dadurch günstiger oder ungünstiger? Dagegen könnte man berechtigt einwenden, dass das Konzept der Institutionalisierungsarbeit anscheinend nur eine Rückkehr zum alten Institutionalismus ${ }^{46}$ oder implizit ein Übergang zur Neuen Institutionenökonomie ist, wo die Vorstellung herrscht, dass Akteure sich die Institutionen schaffen, die sie brauchen, und dass sie die Institutionen verändern, wenn sie nicht mehr passen ${ }^{47}$. Oder man könnte einen erneuten Aufguss von Childs Konzept der Strategic Choice vermuten, das ja bereits die technische Kontingenzebene erweiterte und dem Management politische Einflussnahme in Organisationskontexten zusprach ${ }^{48}$. Auch Vertreter der Actor Network Theory (ANT) könnten einwenden, die Institutional Work-Protagonisten würden zumindest teilweise das Rad neuerfinden, das ANT für sich beansprucht ${ }^{49}$. Insgesamt verwundert, dass Neoinstitutionalisten doch eigentlich behaupten, dass Akteure von Institutionen abhängen und

44 Vgl. hierzu auch Greenwood/Suddaby (2006).

45 Vgl. DiMaggio/Powell (1983), dort der Titel „The iron cage revisited“ sowie die einleitenden Bezüge auf Webers (1978) Die protestantische Ethik und der Geist des Kapitalismus.

$46 \mathrm{Zu}$ „neuem“ und „altem“ Institutionalismus vgl. Selznick (1996). Speziell zum Verhältnis von Institutional Work und ,altem“ Institutionalismus vgl. Kaghan/Lounsbury (2011).

47 Vgl. North (1990), S. 5: „Defining institutions as the constraints that human beings impose on themselves makes the definition complementary to the choice theoretic approach of neoclassical economic theory. ... Institutions are a creation of human beings. They evolve and are altered by human beings; hence our theory must begin with the individual. At the same time, the constraints that institutions impose on individual choices are pervasive."

48 Vgl. Child (1972). In der deutschsprachigen BWL-Literatur ist an dieser Stelle auf Ortmann/Zimmers (1998) „Überlegungen zur strategischen Institutionalisierung und insbesondere rekursiven Regulation“ zu verweisen, die zwar nicht auf Child (1972) aufbauen, aber ebenfalls dem Strategischen Management die Möglichkeiten der Beeinflussung von Recht und Politik aufzeigen, dabei vor einem strukturationstheoretischen Hintergrund argumentieren und so ein Stück weit die praxistheoretische Wende des Neoinstitutionalismus vormachen (siehe Abschnitt 3.3 unten).

49 Diesen Einwand nehmen Lawrence und Suddaby (2006), S. 242ff. bereits vorweg, indem sie ANT als Erweiterungsmöglichkeit für das Konzept der Institutionalisierungsarbeit vorstellen und vorschlagen, ANT-Elemente wie die Umkämpftheit und Übersetzungsnotwendigkeit von Praktiken zu adoptieren sowie (weniger starkt betont) auch die Rolle von Materialität und nicht-menschlichen Aktanten. Für eine Anwendung der ANT in der deutschsprachigen Betriebswirtschaftslehre vgl. etwa Löwer (2005) zum Thema interorganisationale Standards und der zur Etablierung von Standards nötigen, aber dort nicht so bezeichneten, Institutionalisierungsarbeit. 
nicht - wie mit Institutionalisierungsarbeit impliziert - umgekehrt ${ }^{50}$. Ist Institutional Work dann eigentlich noch Neoinstitutionalismus ${ }^{51}$ ?

\subsection{Paradox of Embedded Agency und praxistheoretische Wende}

Institutionalisierungsarbeit findet nicht losgelöst von bestehenden Institutionen und Feldern statt. Wie in Abschnitt 2 oben erläutert, sind Organisationen Teil ihres organisationalen Feldes und können nur aus diesem Feld heraus kreativ, reproduktiv oder destruktiv handeln. Sie stehen also in einer gewissen historisch gewachsenen Beziehung zu dem Sinnsystem des Feldes und zu anderen Organisationen im Feld und handeln vor diesem Hintergrund. Neoinstitutionalisten bezeichnen dies als Einbettung ${ }^{52}$. Wie ist es aber möglich, dass Akteure emanzipiert auf Institutionen einzuwirken versuchen, wenn sie in diese Institutionen regulativ, normativ und kognitiv eingebettet sind ${ }^{53}$ ? Wie kommen sie darauf, dass eine selbstverständliche Regel nicht mehr selbstverständlich sein muss, und woher nehmen sie die Vorstellungskraft, wie die Regel in Zukunft aussehen sollte? Dies ist das sogenannte Paradox of Embedded Agency ${ }^{54}$, mit dem sich viele Neoinstitutionalisten und eben auch die Vertreter des Institutional Work-Ansatzes auseinandersetzen. $\mathrm{Zu}$ der mit dem Konzept der Institutionalisierungsarbeit anvisierten Lösung gelangt man in drei Schritten:

(1) Der neoinstitutionalistische Determinismus wird abgeschwächt, indem man sich wieder bewusst macht, dass Institutionalisierung ein Prozess ist, der niemals endgültig abgeschlossen ist, und dass Institutionalisierung zugleich einen Zustand zu einem Zeitpunkt bezeichnet und somit ein Grad der Institutionalisierung angegeben werden kann; Regeln sind also stets mehr oder weniger, nicht aber absolut institutionalisiert ${ }^{55}$.

(2) Die Handlungsmacht von Akteuren wird ebenfalls relativiert und mehr im Zusammenspiel denn in Konkurrenz mit der Macht von Institutionen gesehen. In oft nicht ganz treffender Bezugnahme auf eine Kritik von DiMaggio ${ }^{56}$ am Ende der 1980er Jahre gängigen Neoinstitutionalismus kam nämlich eine Literatur zum sogenannten Institutional Entrepreneurship auf, in der die Beeinflussbarkeit von Institutionen durch Individuen und Organisationen eher überschätzt wurde ${ }^{57}$. Lawrence und Suddaby wenden sich mit dem Konzept der Institutionalisierungsarbeit explizit gegen die Vorstellung von einzelnen, „hypermuskulären“58 Akteuren, die eigenmächtig Institutionen

50 Vgl. Jepperson (1991); Meyer/Jepperson (2000); Walgenbach (2006), sowie jüngst der Verweis auf die "primacy of institutions" bei Suddaby et al. (2010), S. 1236.

51 Lawrence/Suddaby (2006), S. 219 propagieren explizit keine „return to the rational actor model.“

52 Vgl. zum Beispiel Granovetter (1985); Zukin/DiMaggio (1990).

53 Vgl. Holm (1995).

54 Vgl. Battilana/D’Aunno (2009); Beckert (1999); Garud/Hardy/Maguire (2007); Holm (1995); Seo/Creed (2002); Zietsma/Lawrence (2010).

55 Vgl. DiMaggio (1988), S. 13; Zucker (1977).

56 Vgl. DiMaggio (1988).

57 Vgl. Lawrence/Suddaby/Leca (2009), S. 5. Für einen Überblick zu Institutional Entrepreneurship vgl. Battilanal Leca/Boxenbaum (2009); Garud et al. (2007); Hardy/Maguire (2008); Walgenbach/Meyer (2008b), S. $139 \mathrm{ff}$.

58 Lawrence/Suddaby/Leca (2009), S. 1. 
erschaffen und verändern. Stattdessen wird hervorgehoben, dass zumeist mehrere Akteure - und zwar wohlgemerkt auch die weniger mächtigen - gemeinsam handeln müssten, um eine institutionelle Wirkung zu erreichen, und dass ohnehin ungewiss sei, welche Wirkungen Handlungen haben ${ }^{59}$.

(3) Das Paradox of Embedded Agency soll mithin dahingehend gelöst werden, dass man weder „embeddedness“ noch „agency“ eine Dominanz zuspricht und stattdessen postuliert, dass sie unauflösbar zusammengehören. Um dies theoretisch erfassen zu können, machen die Vertreter des Institutional Work-Ansatzes Anleihen bei der Strukturationstheorie von Giddens und insbesondere bei dessen Konzepten der „knowledgeable agents" und der Dualität von Struktur ${ }^{60}$. Diese Anleihen waren schon 1991 von DiMaggio und Powell angeregt worden ${ }^{61}$, tauchen immer mal wieder im Neoinstitutionalismus auf ${ }^{62}$ und werden dem Konzept der Institutionalisierungsarbeit nun explizit zugrunde gelegt ${ }^{63}$.

Giddens entwickelte eine Theorie, die davon ausgeht, dass Akteure durch ihr Handeln die Bedingungen ihres Handelns produzieren und reproduzieren, sodass diese Bedingungen als Strukturen sowohl Medium als auch Ergebnis des Handelns sind ${ }^{64}$. Im Institutional Work-Ansatz bedeutet dies, dass Institutionalisierungsarbeit sich auf vorhandene Institutionen bezieht, dass Institutionen aber auch durch Institutionalisierungsarbeit erschaffen und entwickelt werden ${ }^{65}$. Wiederum Giddens folgend kommen Lawrence, Suddaby und andere Autoren in der Institutional Work-Literatur des Weiteren zu dem Schluss, dass Handeln und Institutionen durch Praktiken vermittelt werden ${ }^{66}$. Praktiken sind - vereinfachend gesagt - Handlungsmuster, die von einer hinreichenden Zahl von Akteuren regelmäßig angewendet und verstanden werden. Praktiken verknüpfen einzelne Handlungen mit dem größeren Sinnsystem des organisationalen Feldes. Die Institutional Work-Literatur postuliert nun, dass die Erforschung von Institutionalisierungsprozessen sich auf die Erfor-

59 Vgl. explizit Lawrence/Suddaby (2006), S. 217ff.; Lawrence/Suddaby/Leca (2011); außerdem Garud/Jain/Kumaraswamy (2002); Garud/Karnøe (2003); Walgenbach/Meyer (2008a); Wijen/Ansari (2007).

60 Vgl. Giddens (1979) und (1984).

61 Vgl. DiMaggio/Powell (1991), S. 22f.; DiMaggio/Powell (1983), S. 147 beziehen sich bereits in der Einleitung zu ihrem wegweisenden Aufsatz auf Giddens (1979) und dessen Konzept der „structuration.“

62 Vgl. zum Beispiel bei Barley/Tolbert (1997); Beckert (1999); Seo/Creed (2002); Tolbert/Zucker (1996); Walgenbach/Meyer (2008a); Wooten/Hoffman (2008) und nicht zuletzt in Scott (2008). Schiller-Merkens (2008) entwirft eine strukturationstheoretische Konzeption von institutionellem Wandel; Walgenbach/Meyer (2008b), S. 131ff. unternehmen ebenfalls einen „Verknüpfungsversuch“ zwischen Neoinstitutionalistischer Organisationstheorie und Strukturationstheorie.

63 Vgl. Lawrence/Suddaby (2006), S. 218ff.: „Our concept of institutional work follows in this practice tradition: we view institutional work as intelligent, situated institutional action." Giddens steht dabei im Vordergrund, wobei auch auf andere Vertreter einer "Sociology of Practice“ verwiesen wird, insbesondere auf Bourdieu und de Certeau. Zusammenfassend: „The emergence of practice theory provides a theoretical foundation for understanding institutional work“, Lawrence/Suddaby (2006), S. 246; vgl. auch Kraatz (2011); Lawrence/Suddaby/Leca (2011); Zietsma/Lawrence (2010), S. 191.

64 Vgl. Giddens (1979), S. 5 und (1984), S. $25 \mathrm{ff}$. und 374.

65 Vgl. zu dieser Rekursivität bereits Barley/Tolbert (1997).

66 Vgl. Lawrence/Suddaby (2006), S. $218 \mathrm{ff}$. 
schung von Praktiken der Institutionalisierungsarbeit konzentrieren sollte ${ }^{67}$. So läuft es letztlich darauf hinaus, dass mit dem Fokus auf Institutionalisierungsarbeit der Neoinstitutionalismus zu einer Praxistheorie wird oder zumindest einen praxistheoretischen Zweig entwickelt (siehe Abbildung 2).

\section{Abbildung 2: Institutionalisierungsarbeit als Praktiken in einem Organisationalen Feld (Schematische Darstellung)}

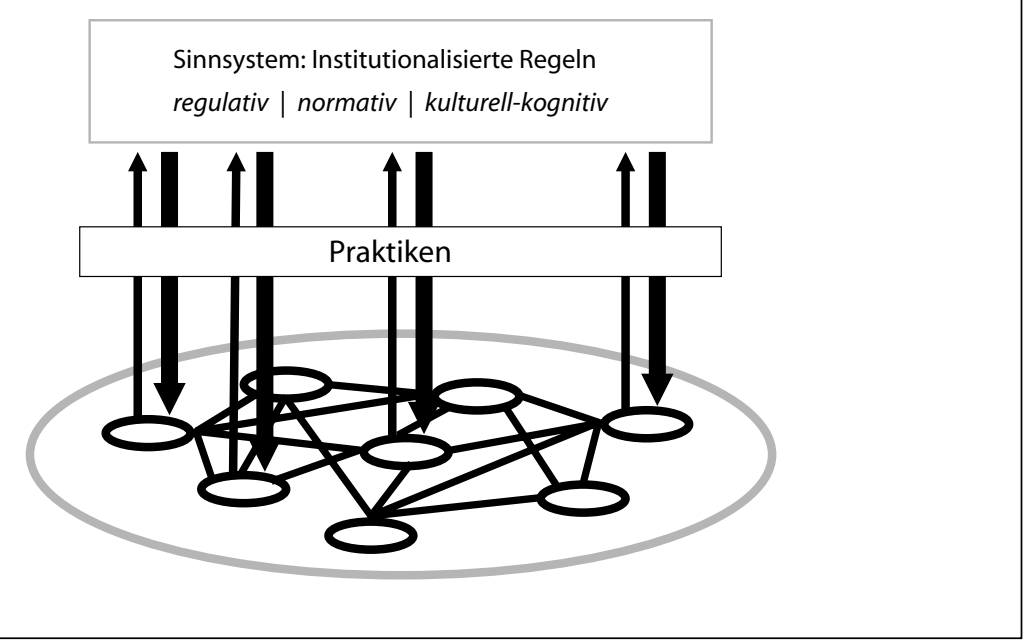

\subsection{Praktiken der Institutionalisierungsarbeit}

Ein großer Vorteil dieser praxistheoretischen Rahmung von Institutionalisierungsarbeit besteht darin, dass spezifische Praktiken identifiziert werden können, die im anderen Sinne des Wortes „praktisch“ sind, weil sie zeigen, wie Institutionalisierungsarbeit aussieht und welche verschiedenen Formen es gibt. Lawrence und Suddaby haben explorativ die von 1990 bis 2005 in Administrative Science Quarterly, Academy of Management Journal und Organization Science veröffentlichten, empirischen Studien der neoinstitutionalistischen Forschung ausgewertet und daraus einen vorläufigen Katalog der vielfältigen Praktiken von Institutionalisierungsarbeit abgeleitet ${ }^{68}$. Für das Erschaffen von Institutionen

67 Vgl. Lawrence/Suddaby (2006), S. 220; Lawrence/Suddaby/Leca (2009), S. 14. Die praxistheoretische Wendung wird dadurch komplettiert, dass Praktiken der Institutionaliserungsarbeit auf die im Feld institutionalisierten oder zu institutionalisierenden Praktiken gerichtet sind, es also um Praktiken der Beeinflussung von Praktiken geht (vgl. Zietsma/Lawrence (2010)).

68 Vgl. Lawrence/Suddaby (2006), S. 220ff. Diese Studien stehen zumeist nicht explizit unter dem Label Institutional Work, zumal sie meistens schon lange, bevor dieses Label (wieder) eingeführt wurde, veröffentlicht wurden. Es handelt sich also um eine rückwirkende Zuordnung empirischer Studien, in denen es unter Bezugnahme auf den Neoinstitutionalismus um die Beeinflussung von Institutionen geht, zu einem neuen Literaturstrang. 
gibt es die Praktiken Advocacy, Defining, Vesting und weitere bis hin zu Theorizing und Educating. Für das Erhalten von Institution gibt es: Enabling, Policing et cetera bis hin zu Routinizing. Und für das Stören von Institutionen haben Lawrence und Suddaby immerhin drei empirisch belegte Praktiken gefunden: Disconnecting sanctions, Disassociating moral foundations und Undermining assumptions and beliefs. Dahinter stehen jeweils empirische Fälle, in denen diese Praktiken gelebt wurden, und so kann der Katalog mit ein wenig Übersetzungshilfe meines Erachtens bereits von Nutzen für Entscheider in Unternehmen sein, die die Institutionen in ihrem organisationalen Feld beeinflussen wollen. Zugleich ist der Katalog eine Vorlage für alle Forscher, die in einem bestimmten organisationalen Feld Praktiken der Institutionalisierungsarbeit identifizieren möchten. Dabei ist die Anschlussfähigkeit dieses Katalogs allerdings ein wenig fragwürdig, da allgemein bekannte Praktiken der Umweltbeeinflussung durch Unternehmen - vor allem das Lobbying - in dem Katalog von Lawrence und Suddaby nicht vorkommen, sondern quer zur Systematik des Erschaffens, Erhaltens und Erschütterns von Institutionen zu liegen scheinen und sich eher durch den Adressaten der Institutionalisierungsarbeit charakterisieren lassen (im Falle des Lobbying wären dies staatliche oder andere übergeordnete Regulierungsorgane).

\subsection{Kritik am Institutional Work-Ansatz}

Bevor im nächsten Abschnitt das Konzept der feldkonfigurierenden Veranstaltungen vorgestellt wird, das in engem Zusammengang zur Fokussierung auf Praktiken im Institutional Work-Ansatz zu sehen ist, werde ich einige Schwachpunkte der bisherigen Konzeptualisierung von Institutionalisierungsarbeit hervorheben:

(1) Es ist noch unklar, ob die Abkehr vom Determinismus und von der starken Dominanz der Institutionen in der Neoinstitutionalistischen Organisationstheorie nachhaltig ist. Die Neoinstitutionalisten diskutieren, ob sich Institutional Work nicht zu weit vom Kern der Theorie entfernt ${ }^{69}$. Es könnte also sein, dass die vielversprechenden Schritte, die getan wurden, wieder revidiert werden oder aber dass die praxistheoretische Untersuchung von Institutionalisierungsarbeit nur eine Nische abseits des neoinstitutionalistischen Mainstreams ausfüllt ${ }^{70}$.

(2) Mit der praxistheoretischen und speziell strukturationstheoretischen Fundierung handelt sich der Institutional Work-Ansatz auch Probleme ein, die mit derartigen Sozialtheorien einhergehen. Insbesondere scheint das Konzept der Institutionalisierungsarbeit bisher vor allem einen deskriptiven Nutzen zu haben. Man hat zwar empirisch zeigen können, dass die Beeinflussung von Institutionen auf vielfältige Weise möglich ist ${ }^{71}$. Man ist jedoch weit davon entfernt, prognostizieren zu können, wann Institutionalisierungsar-

69 Vgl. Hwang/Colyvas (2011); Kaghan/Lounsbury (2011); Kraatz (2011); Phillips (2011). Senge (2011), S. 163 gibt kritisch zu bedenken: „Versucht man hingegen allen theoretischen Positionen gerecht zu werden, steht zu befürchten, dass von dem eigentlichen neo-institutionalistischen Kern nicht mehr viel übrig bleibt."

70 Vgl. zum Beispiel Walgenbach (2006), S. 391, der vor Widersprüchen warnt, wenn der Neoinstitutionalismus vom Paradigma der institutionellen Bestimmtheit von Akteuren abweicht.

71 Vgl. Lawrence/Suddaby (2006), S. 220ff. sowie neue empirische Fallstudien von Rojas (2010) und Zietsma/Lawrence (2010). 
beit tatsächlich Wirkungen zeitigt oder welche Formen von Institutionalisierungsarbeit unter welchen Umständen zu erwarten $\operatorname{sind}^{72}$. Damit bleibt unklar, wann es sich für Unternehmen lohnt, in Institutionalisierungsarbeit zu investieren. Dies entspricht der Kritik an der Strukturationstheorie, dass sie zwar einen heuristischen Wert habe, aber kaum zur Generierung und Überprüfung von Hypothesen geeignet sei ${ }^{73}$.

(3) Aus der Sicht einer Organisation in einem organisationalen Feld scheint das Paradox of Embedded Agency keineswegs gelöst oder es müsste zumindest eine Theorie der Kreativität oder des Entrepreneurship hinzukommen, um zu erfassen, wie Akteure sich an einem Sinnsystem orientieren und es zugleich womöglich verändern wollen ${ }^{74}$.

(4) Noch unbefriedigender ist die Situation aus der Sicht von Organisationen, die sich in einem neu entstehenden, also noch nicht gefestigten organisationalen Feld befinden. Auf welcher Grundlage sollen sie angesichts großer Ungewissheit und Ambiguität „absichtsvoll“ handeln ${ }^{75}$ ? Haben sie etwa doch vom Sinnsystem unabhängige Interessen; oder orientieren sie sich ganz isomorph an anderen, früheren, benachbarten Feldern?

Ein wichtiger nächster Schritt in der Systematisierung und theoretischen Ausdifferenzierung von Institutionalisierungsarbeit könnte sein, das Konzept (wieder) stärker an Scotts Begriff der organisationalen Felder und dessen Drei-Säulen-Modell anzubinden (siehe Abschnitt 2 oben). Das hieße zunächst, dass man die drei Formen der Institutionalisierungsarbeit als Praktiken des Erschaffens, Erhaltens und Erschütterns von Institutionen zu den regulativen, normativen und kulturell-kognitiven Säulen in Scotts Model in Beziehung setzt. Die Leitfrage wäre, ob die drei Säulen je unterschiedlich stark angesprochen werden (sollten), je nachdem, ob das Erschaffen, Erhalten oder Erschüttern einer Institution beabsichtigt ist ${ }^{76}$. Dies ist insbesondere vor dem Hintergrund interessant, dass

72 Vgl. Lawrence/Suddaby (2006), S. 228: „As with all our observations in this section, we draw our insights ... from a small sample of research, primarily case studies, and have been unable to gauge their generalizability." Hwang/Colyvas (2011), S. 64 bemängeln: „It is not clear which types of institutions are more susceptible for different kinds of institutional work. "Zietsma/Lawrence (2010) liefern zumindest erste empirisch fundierte Ansätze für verallgemeinerbare Erklärungen des Auftretens bestimmter Formen von Institutionalisierungsarbeit unter bestimmten Umständen.

73 Hierbei ist wenig hilfreich, dass Giddens (1984), S. 326f. selber mit den Begriffen seiner Strukturationstheorie nicht viel mehr beansprucht, als dass „they may be useful for thinking about research problems and the interpretation of research results."

74 Lawrence/Suddaby (2006), S. 220 bleiben beim Postulat der Einbettung und halten daran fest, dass „we cannot step outside of action as practice." Dass institutionelle Innovationen dennoch von Akteuren ausgehen, bleibt hiermit vor allem eine weiter zu untersuchende Behauptung. Erste Ansätze finden sich in der Institutional WorkLiteratur in Form der Anwendung der Agency-Dimensionen von Emirbayer/Mische (1998) insbesondere bei Battilana/D'Aunno (2009) und bereits bei Dorado (2005), vgl. auch Lawrence/Suddaby/Leca (2011). Willmott (2011), S. 67 kritisiert allerdings: „Current proposals to pay closer attention to 'institutional work' are seen to reproduce the dualism of individual (agency) / institution (structure) as they invite a further flip-flop between these poles."

75 Vgl. Beckert (1999); Möllering (2010). Hierzu auch Hardy/Maguire (2008), S. 203: „The exact nature of the relationship between uncertainty and institutional entrepreneurship is, therefore, not clear, and further research is warranted."

76 Vgl. Battilana/D’Aunno (2009), S. 47ff., die eine ähnliche Matrix anregen, allerdings nicht mit Scotts drei Säulen, sondern mit Emirbayer/Misches (1998) drei Dimensionen von Agency. 
ja ein Teil der Organisationen eines Feldes eine Veränderung von Institutionen beabsichtigen könnten, während andere Organisationen zugleich die bestehenden Institutionen erhalten wollen ${ }^{77}$. Womöglich nehmen diese Akteure unterschiedliche Säulen ins Visier oder aber ihre Praktiken der Institutionalisierungsarbeit treffen typischer Weise in bestimmten Säulen aufeinander. Tabelle 1 zeigt exemplarisch eine entsprechende 3x3Matrix von Institutional Work-Formen und institutionellen Säulen, die eine systematischere und differenziertere Forschung zu Institutionalisierungsarbeit anleiten könnte.

\section{Tabelle 1: Exemplarische Matrix der institutionellen Säulen (nach Scott (2008)) und der Formen von Institutionalisierungsarbeit (nach Lawrence/Suddaby (2006))}

\begin{tabular}{|l|c|c|c|}
\hline $\begin{array}{l}\text { Formen der Institutio- } \\
\text { nalisierungsarbeit }\end{array}$ & $\begin{array}{c}\text { Regulative } \\
\text { Säule }\end{array}$ & $\begin{array}{c}\text { Normative } \\
\text { Säule }\end{array}$ & $\begin{array}{c}\text { Kulturell- } \\
\text { kognitive Säule }\end{array}$ \\
\hline $\begin{array}{l}\text { Erschaffen von } \\
\text { Institutionen }\end{array}$ & $\begin{array}{c}\text { Gesetzgebung } \\
\text { beeinflussen }\end{array}$ & $\begin{array}{c}\text { Neue Werte } \\
\text { propagieren }\end{array}$ & $\begin{array}{c}\text { Ausbildungswege } \\
\text { neu einführen }\end{array}$ \\
\hline $\begin{array}{l}\text { Erhalten von } \\
\text { Institutionen }\end{array}$ & $\begin{array}{c}\text { Regelverstöße } \\
\text { rechtlich ahnden }\end{array}$ & $\begin{array}{c}\text { "Best Practices" } \\
\text { belohnen }\end{array}$ & $\begin{array}{c}\text { Traditionen und } \\
\text { Rituale pflegen }\end{array}$ \\
\hline $\begin{array}{l}\text { Erschüttern von } \\
\text { Institutionen }\end{array}$ & $\begin{array}{l}\text { Grauzonen und } \\
\text { Lücken ausnutzen }\end{array}$ & $\begin{array}{c}\text { Externalitäten } \\
\text { skandalisieren }\end{array}$ & $\begin{array}{c}\text { Fremde und } \\
\text { Fremdes zeigen }\end{array}$ \\
\hline
\end{tabular}

Quelle: eigene Beispiele

Die Kritik und die Erweiterungsmöglichkeiten zum Konzept der Institutionalisierungsarbeit sind hiermit keineswegs erschöpft. Man muss den Vertretern des Ansatzes zugestehen, dass sie offen mit Einwänden und Desideraten umgehen ${ }^{78}$ und sich zum Beispiel jüngst in einem „Dialog“ im Journal of Management Inquiry " der Diskussion stellen. Die große Offenheit, die dabei an den Tag gelegt wird, um den Einklang von Institutional Work nicht nur mit der Strukturationstheorie, sondern auch anderen Strömungen wie etwa der Actor Network Theory herzustellen, lässt dabei allerdings den neoinstitutionalistischen Kern und originären Beitrag des Konzeptes verschwimmen. Aus Sicht der Betriebswirtschaftslehre ist dennoch zu begrüßen, dass das Konzept der Institutionalisierungsarbeit sich zu der aktiven Rolle von Individuen und Organisationen in Prozessen der Entstehung und Entwicklung von organisationalen Feldern bekennt ${ }^{80}$ und dass es das Augenmerk von Forschung und Praxis eben auf nachvollziehbare Praktiken lenkt. Zu diesem Ansatz passt gut das etwas speziellere Konzept der feldkonfigurierenden Veranstaltungen, das ich nun als nächstes betrachte.

77 Vgl. auch Zietsma/McKnight (2009).

78 Bereits in Lawrence/Suddaby (2006), S. 246 wird eingestanden: „Large gaps exist in our ability to describe institutional work, let alone explain it."Vgl. auch Lawrence/Suddaby/Leca (2009).

79 Vgl. Journal of Management Inquiry, Vol. 20, Heft 1 (2011).

80 Lawrence/Suddaby/Leca (2011), S. 53: „The concept of institutional work highlights the (perhaps controversial) notion that individuals actively engage in processes of institutional creation, maintenance, disruption, and change." 


\section{Feldkonfigurierende Veranstaltungen}

\subsection{Institutionalisierungsarbeit in Aktion?}

Wenn man das Konzept der Institutionalisierungsarbeit ernst nimmt, dann fragt sich, wie und wo man Praktiken der Institutionalisierungsarbeit empirisch erfassen kann. Ein vielversprechender Ansatz hierzu steckt im Konzept der Field-Configuring Events ${ }^{81}$, womit zunächst pauschal Veranstaltungen gemeint sind, bei denen Felder konfiguriert werden. Feldkonfiguration wiederum bedeutet für organisationale Felder, wie in Abschnitt 2 dargestellt, dass ein Sinnsystem und die Feldzugehörigkeit von Organisationen etabliert werden.

Das methodische Problem ist, dass es nicht ausreicht, ein einzelnes Unternehmen zu beobachten, wie es auf Institutionen reagiert und versucht, sie zu beeinflussen. Oliver hat zwar bereits 1991 einen Katalog von strategischen Optionen aufgestellt, die einem Unternehmen im Umgang mit Institutionen zur Verfügung stehen ${ }^{82}$. Und Aldrich und Fiol haben 1994 in einem Aufsatz aufgelistet, mit welchen Maßnahmen Unternehmen Legitimität für die Erschaffung eines neuen Feldes produzieren können, darunter auf der institutionellen Ebene unter anderem auch das Organisieren kollektiver Marketing- und Lobbying-Anstrengungen ${ }^{83}$. Doch auch diese beiden Ansätze, die zunächst fragen, was das einzelne Unternehmen tun kann, erfordern in der empirischen Forschung die parallele Beobachtung einer Vielzahl von Unternehmen, die miteinander interagieren. Man müsste darüber hinaus einen Weg finden zu zeigen, wie sich das Sinnsystem verändert. Schon aus diesen forschungspraktischen Gründen ist es interessant, Orte und Zeitpunkte zu identifizieren, an denen Institutionalisierungsarbeit besonders intensiv ist und an denen ein organisationales Feld besonders geprägt wird ${ }^{84}$. Organisationsforscher, die in diese Richtung arbeiten, sprechen seit einiger Zeit von den bereits mehrfach erwähnten feldkonfigurierenden Veranstaltungen (Field-Configuring Events) und spätestens seit einem Sonderheft des Journal of Management Studies $^{85}$ in 2008 ist hierzu eine eigene Forschungsströmung entstanden.

\subsection{Zum Begriff der feldkonfigurierenden Veranstaltung}

Lampel und Meyer bezeichnen in ihrem zentralen konzeptionellen Artikel Field-Configuring Events als ,temporary social organizations such as tradeshows, professional gatherings, technology contests, and business ceremonies that encapsulate and shape the development of professions, technologies, markets, and industries " 86 . Veranstaltungen wie Kongresse und Messen zeigen demnach einen „sozialen Mikrokosmos“87, an dem man die Entwicklung des organisationalen Feldes als Makrokosmos studieren kann. Wenn wir uns auf Scotts Definition

81 Dieser Bezug wurde jüngst auch explizit von Hardy/Maguire (2010) hergestellt, die ihre Studie einer feldkonfigurierenden Veranstaltung auf das Konzept der Institutionalisierungsarbeit beziehen.

82 Vgl. Oliver (1991).

83 Vgl. Aldrich/Fiol (1994), S. 659ff.; vgl. auch Suchman (1995) und Lawrence (1999).

84 Vgl. Lampel/Meyer (2008), S. 1030.

85 Journal of Management Studies, Vol. 45, Heft 6 (2008).

86 Lampel/Meyer (2008), S. 1026; vgl. in Ansätzen bereits Meyer/Gaba/Colwell (2005).

87 Lampel/Meyer (2008), S. 1030. 
des organisationalen Feldes ${ }^{88}$ besinnen, ist dieser Ansatz einleuchtend. Durch die Teilnahme an der Veranstaltung wird signalisiert, wer sich selbst dem Feld zugehörig fühlt. Bei den Veranstaltungen interagieren Organisationen des gleichen Feldes besonders intensiv. Und bei den Veranstaltungen beziehen sie sich auf das gemeinsame Sinnsystem und können es zugleich hinterfragen, interpretieren, erweitern, umdeuten und so weiter (siehe Abbildung 3). Feldkonfigurierende Veranstaltungen bieten mithin Gelegenheiten für Institutionalisierungsarbeit ${ }^{89}$.

\section{Abbildung 3: "Verdichtung" des Organisationalen Feldes bei Events (Schematische Darstellung)}

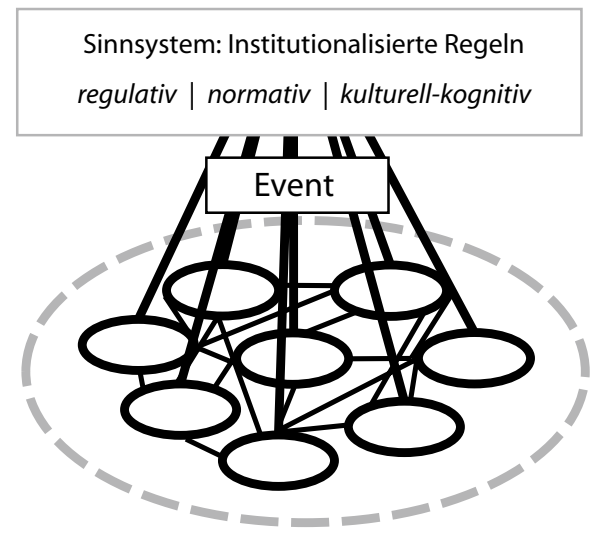

Wohlgemerkt sind Events als Veranstaltungen zu verstehen und nicht als Ereignisse aller $\mathrm{Art}^{90}$. Events sind geplant und organisiert und bereits das impliziert Institutionalisierungsarbeit $^{91}$. Im Gegensatz zu den „small events“92 in der Pfadtheorie sind es eher „big events“, zumindest in der Intention der Organisatoren ${ }^{93}$. Was bei Events passiert, ist freilich nicht völlig planbar und vorhersehbar. Es kann bei ihnen scheinbar nebensächliche, unbedeu-

88 Vgl. Scott (1994), siehe Abschnitt 2 oben.

89 Hierbei spielt gerade vor dem Hintergrund des Paradox of Embedded Agency auch eine wichtige Rolle, dass nicht nur Organisationen, sondern ihre Vertreter als Individuen außerhalb des üblichen Organisationskontextes (kreativer?) miteinander interagieren können, vgl. Lampel/Meyer (2008), S. 1028.

90 Das englische Wort „event“ hat grob genommen drei unterschiedliche Bedeutungen: Ereignis, Veranstaltung und Fall. Die zweite Bedeutung ist bei Field-Configuring Events gemeint. Das (oder der) Event ist im Übrigen längst auch ein deutsches, im Duden geführtes Wort mit der gleichen - bisweilen dramatisch aufgeladenen - Bedeutung wie Veranstaltung.

91 Vgl. Lampel/Meyer (2008), S. 1026: „Organizers often design FCEs with an eye toward influencing field evolution. Organizers' intentions are not always realized, of course." Hierzu passend die praxistheoretische Fundierung bei Lawrence/Suddaby (2006), S. 219: „A practice theory describes the intelligent activities of individuals and organizations who are working to effect those events and outcomes."

92 Arthur (1994), S. 14.

93 Vgl. zu „bigger events“ als mögliche Auslöser von Pfadabhängigkeit Sydow/Schreyögg/Koch (2009). 
tende Vorkommnisse geben, die erst später als „critical juncture“94 in ihrer nachhaltigen Bedeutung für die Feldentwicklung erkennbar werden.

Als ein Beispiel für feldkonfigurierende Veranstaltungen können Preisverleihungen dienen, etwa Innovationspreise für Unternehmen, mit denen wünschenswertes Verhalten innerhalb des Feldes belohnt und zugleich Innovativität als Ideal gefestigt wird. Die Einführung neuer Preiskategorien, zum Beispiel eines Ökologie-Innovationspreises, propagiert oder dokumentiert eine Erweiterung des Sinnsystems, in diesem Beispiel um ökologische Kategorien und Werte ${ }^{95}$. Die dem Feld angehörenden Unternehmen sind dann nicht innovativ oder umweltfreundlich, weil sie die Auszeichnung bekommen könnten ${ }^{96}$, sondern die Ausschreibung des Preises signalisiert allgemeiner, welches Verhalten im Feld legitimiert ist. Einschlägige Studien hierzu gibt es etwa von Anand und Kollegen über den Booker Prize ${ }^{97}$ und die Grammy Awards $^{98}$. Gerade die sogenannten Creative Industries bieten ein weites und zunehmend beachtetes Feld für die organisationstheoretische Untersuchung von Events, wie ein kürzlich erschienener Band von Moeran und Strandgaard Pedersen eindrucksvoll belegt ${ }^{99}$. Andere Studien, wie etwa die von Garud über Cochleaimplantate, zeigen, wie auf Kongressen wichtige Weichen gestellt werden zum Beispiel hinsichtlich der in einem Feld dominant verwendeten Technologie ${ }^{100}$. Ähnliches untersuche ich selbst empirisch für die Halbleiterindustrie, wo die weitere Entwicklung des Feldes oder gar das Entstehen eines neuen Feldes von Richtungsentscheidungen abhängt, die auf Fachkongressen diskutiert werden ${ }^{101}$. Unternehmen versuchen bei solchen Veranstaltungen, ihre Interessen durchzusetzen ${ }^{102}$. Sie nehmen aber vor allem auch an diesen Veranstaltungen teil, um erst einmal herauszufinden, worum es geht, welche Optionen es gibt und was die eigenen Interessen eigentlich sind ${ }^{103}$.

\subsection{Merkmale von feldkonfigurierenden Veranstaltungen}

Lampel und Meyer definieren sechs Kriterien, die charakteristisch für feldkonfigurierende Veranstaltungen sind (eigene Übersetzung): (1) Sie versammeln Akteure aus verschiedenen professionellen, organisationalen und geografischen Hintergründen an einem Ort; (2) die Dauer der Veranstaltungen ist begrenzt, meist auf wenige Stunden oder Tage; (3) sie bieten unstrukturierte Gelegenheiten zur persönlichen, sozialen Interaktion und (4) beinhalten auch zeremonielle und dramaturgische Aktivitäten; (5) sie geben Gelegenheiten zum Informati-

94 Vgl. Collier/Collier (1991).

95 Ein Beispiel wäre der Preis „Der Gelbe Engel“ in der Kategorie Innovation und Umwelt, der seit 2005 vom Allgemeinen Deutschen Automobil Club (ADAC) verliehen wird.

96 Das bedeutet natürlich nicht, dass die Preisträger sich nicht über Preisgelder oder mit dem Preis verbundene zusätzliche Aufmerksamkeit und Umsätze freuen.

97 Vgl. Anand/Jones (2008).

$98 \mathrm{Vgl}$. Anand/Watson (2004).

99 Vgl. Moeran/Strandgaard Pedersen (2011).

100 Vgl. Garud (2008).

101 Vgl. Möllering (2010).

102 Vgl. Lampel/Meyer (2008), S. 1030: „Field configuring events are often championed and organized by institutional entrepreneurs with an overt field-building agenda, but also with a view towards private gains."

103 Vgl. hierzu auch Zilber (2007). 
onsaustausch und zur kollektiven Sinnstiftung; (6) und sie bringen soziale und reputationsrelevante Ressourcen hervor, die woanders und für andere Zwecke eingesetzt werden können ${ }^{104}$.

Jeder Kongress, jede Messe, jede Preisverleihung etc. könnte nun daraufhin untersucht werden, ob diese Punkte erfüllt sind. Tabelle 2 zeigt dies am Beispiel eines von mir untersuchten Kongresses in der Halbleiterindustrie, dem Fifth Sematech Workshop on Next Generation Lithography in Pasadena, 28.-30. August $2001^{105}$. Die linke Spalte bildet die sechs Kriterien von Lampel und Meyer ab und die rechte Spalte bestätigt deren prinzipielle Anwendbarkeit auf die Veranstaltung in Pasadena. Auf diese Weise könnten Veranstaltungen mit feldkonfigurierendem Potenzial identifiziert werden und man könnte sodann genauer schauen, welche Institutionalisierungsarbeit dort konkret geleistet wird. Das kann interessante Erkenntnisse bringen. Der Ansatz hat jedoch in der bisher vorliegenden Ausarbeitung noch erhebliche Schwachpunkte, von denen ich im Folgenden die wichtigsten benennen möchte.

\section{Tabelle 2: Anwendung der Charakteristika feldkonfigurierender Veranstal- tungen auf das Beispiel des Fifth NGL Workshops, Pasadena (2001)}

\begin{tabular}{|c|c|}
\hline $\begin{array}{c}\text { Characteristics of Field-Configuring } \\
\text { Events (Lampel/Meyer 2008) }\end{array}$ & $\begin{array}{l}\text { Characteristics of the } \\
\text { Fifth NGL Workshop }\end{array}$ \\
\hline $\begin{array}{l}\text { 1. FCEs assemble in one location actors from } \\
\text { diverse professional, organizational, and } \\
\text { geographical backgrounds. }\end{array}$ & $\begin{array}{l}\text { The conference in a hotel in Pasadena assembled } \\
\text { participants coming from a broad range of } \\
\text { engineering disciplines and business functions: } \\
\text { from manufacturers, suppliers, R\&D centers, and } \\
\text { consortia, and from North America, Asia, and Europe. }\end{array}$ \\
\hline $\begin{array}{l}\text { 2. FCEs' duration is limited, normally running } \\
\text { from a few hours to a few days. }\end{array}$ & $\begin{array}{l}\text { The conference lasted two days plus some pre- and } \\
\text { post-conference meetings. }\end{array}$ \\
\hline $\begin{array}{l}\text { 3. FCEs provide unstructured opportunities for } \\
\text { face-to-face social interaction. }\end{array}$ & $\begin{array}{l}\text { The conference included three receptions, a poster } \\
\text { session, breakfast, lunches and coffee breaks, and } \\
\text { the conference dinner; there was time for private } \\
\text { dinner arrangements, and informal meetings could } \\
\text { be held alongside the conference program. }\end{array}$ \\
\hline $\begin{array}{l}\text { 4. FCEs include ceremonial and dramaturgical } \\
\text { activities. }\end{array}$ & $\begin{array}{l}\text { The conference was held at the rather luxurious } \\
\text { and glamorous Ritz-Carlton Huntington Hotel, } \\
\text { included a closing dinner with survey results as its } \\
\text { grand finale, and had a carefully planned agenda } \\
\text { featuring the so-called Technical Champions. }\end{array}$ \\
\hline $\begin{array}{l}\text { 5. FCEs are occasions for information exchange } \\
\text { and collective sense-making. }\end{array}$ & $\begin{array}{l}\text { The conference program consisted mainly of presen- } \\
\text { tations for exchanging information on progress in } \\
\text { NGL technology; the survey exercise at the end gave } \\
\text { a collective picture of opinions within the field. }\end{array}$ \\
\hline $\begin{array}{l}\text { 6. FCEs generate social and reputational } \\
\text { resources that can be deployed elsewhere and } \\
\text { for other purposes. }\end{array}$ & $\begin{array}{l}\text { The conference generated many resources, the most } \\
\text { significant one being the Final Report and, more } \\
\text { importantly, the survey results distributed after the } \\
\text { conference, in the form of presentation slides that } \\
\text { would be used to push the case for technological } \\
\text { options at later events. }\end{array}$ \\
\hline
\end{tabular}

Quelle: Möllering (2010), S. 14f., Table 1.

104 Vgl. Lampel/Meyer (2008), S. 1027.

105 Vgl. Möllering (2010), S. $14 \mathrm{f}$. 


\subsection{Kritik am Field-Configuring Event-Ansatz}

Erstens ist das Konzept der feldkonfigurierenden Veranstaltungen entweder tautologisch oder unterspezifiziert hinsichtlich der Wirkungen von Veranstaltungen. Tautologisch hieße, man bezeichnet eine Veranstaltung ex post als „field configuring“, wenn sie in ihrer Wirkung tatsächlich ein Feld konfiguriert hat. Blendet man damit alle anderen Veranstaltungen aus, die keine oder nur eine schwache Konfigurationswirkung haben? Der Kriterienkatalog scheint eher geeignet, potenziell feldkonfigurierende Veranstaltungen zu identifizieren $^{106}$. Warum einige Veranstaltungen eine größere Wirkung haben als andere, bleibt noch unklar, vor allem ex ante. An welchen Veranstaltungen soll sich ein Unternehmen beteiligen, weil sie wichtige Wirkungen haben könnten, und an welchen nicht?

Zweitens, in engem Zusammenhang mit dem vorigen Punkt, steht das Problem, dass die Literatur zu feldkonfigurierenden Veranstaltungen nur eine rudimentäre Theorie des Events enthält. Diese müsste ausgearbeitet werden. Etwa: Warum lassen sich gewisse Wirkungen erzielen, wenn Akteure auf engem zeitlichem und geografischem Raum interagieren und dabei auch Zeremonien und Rituale durchführen? Warum haben Events eine bindende Kraft? Hier könnten wohl anthropologische Studien über Rituale ${ }^{107}$ hilfreich sein. Dacin, Munir und Tracey zeigen in einer neuen Studie über das „Formal Dining at Cambridge Colleges“ aus einer neoinstitutionalistischen Perspektive, welche Rolle Rituale beim Erhalten und Fortschreiben von Institutionen spielen können ${ }^{108}$. Dies müsste auf Rituale bei Veranstaltungen ausgeweitet werden, die dem Erschaffen oder Erschüttern von Institutionen dienen sollen. Ein besseres Verständnis von Inszenierung, Interaktionen und Ritualen bei Veranstaltungen könnte auch auf die Arbeiten von Goffman aufbauen ${ }^{109}$. Außerdem könnten man Anleihen bei entscheidungstheoretischen Erkenntnissen zu kollektiven Entscheidungen unter Zeitdruck und Ungewissheit bei begrenzten, eher zufällig gegebenen Ressourcen machen, etwa dem wohlbekannten Garbage Can-Modell ${ }^{110}$. Aus alledem ergeben sich Hinweise, wie eine wirkungsvolle feldkonfigurierende Veranstaltung organisiert sein müsste. Der Kriterienkatalog von Lampel und Meyer suggeriert eine Mischung aus Offenheit und Raum für spontane Interaktion einerseits sowie Abgeschlossenheit und feste Skripte andererseits. In einem kürzlich erschienenen Buch-Beitrag sagt Lampel, es ginge darum, einen Raum für ,predictable unpredictability “ $\mathrm{zu}_{\text {schaffen }}{ }^{111}$. Was ist jedoch empirisch jeweils die richtige Mischung, damit Veranstaltungen nicht zu unverbindlich und überrschend aber auch nicht zu einengend und vorhersehbar für die Teilnehmer sind? Und geht es vielleicht nicht nur um einzelne Events, sondern um die Veranstaltung einer ganzen Serie von Events ${ }^{112}$ ?

Drittens ist die Konzentration auf Veranstaltungen als Mikrokosmen nur scheinbar eine methodische Erleichterung, denn jedes Event kann nur eine Episode in einem größeren Prozess sein ${ }^{113}$. Lampel und Meyer schreiben selbst: „Field configuring events are both the

106 Vgl. Möllering (2010), S. 11.

107 Vgl. zum Beispiel Douglas (1970).

108 Vgl. Dacin/Munir/Tracey (2010).

109 Vgl. Goffman (1959), S. 1967.

110 Vgl. zum Garbage Can-Modell (Mülleimer-Modell) Cohen/March/Olsen (1972).

111 Lampel (2011), S. 342ff.

112 Vgl. Dobusch/Müller-Seitz (2011).

113 Vgl. Hardy/Maguire (2010). 
products and the drivers of field evolution" ${ }^{114}$. Das klingt einerseits konsequent prozesstheoretisch und passt gut zu einem strukturationstheoretisch erweiterten Neoinstitutionalismus, der die Dualität von Struktur ernst nimmt. Andererseits kommt man demnach nicht ohne Methoden aus, die Makrostrukturen erfassen können, wie etwa die über einzelne Veranstaltungen hinausgehende Netzwerkanalyse oder Diskursanalyse.

Das Konzept der feldkonfigurierenden Veranstaltungen sollte mithin noch nachdrücklicher die bei Veranstaltungen geleistete Institutionalisierungsarbeit praxistheoretisch verstehen und zugleich, wie bereits am Ende von Abschnitt 3 für die weitere Entwicklung der Forschungen zu Institutionalisierungsarbeit angeregt, einen stärkeren Bezug zu dem in Abschnitt 2 dargestellten Konzept des organisationalen Feldes herstellen. Oben (Abschnitt 3.5, Tabelle 1) habe ich erste Überlegungen zu einer 3x3-Matrix angestellt, welche die drei Formen der Institutionalisierungsarbeit (Erschaffen, Erhalten und Erschüttern von Institutionen) in Beziehung zu den drei institutionellen Säulen (regulative, normative und kulturell-kognitive Institutionen) von Scott setzt. Der Field-Configuring Events-Ansatz kann sich weiterentwickeln und zugleich zum Institutional Work-Ansatz beitragen, indem er für jede der neun Zellen der Matrix einschlägige Veranstaltungen identifiziert. Welche Art von Veranstaltung unterstützt etwa in besonderer Weise das regulative Erschaffen einer neuen Institution, welche Art von Veranstaltungen dient eher dem normativen Erhalten von Institutionen, und welche Art von Veranstaltungen kann die kulturell-kognitive Basis von Institutionen erschüttern? Die Schwierigkeit einer solchen Zuordnung besteht allerdings darin, dass viele Veranstaltungsformen, wie etwa Konferenzen, Preisverleihungen oder Messen, im Prinzip für alle drei Formen der Institutionalisierungsarbeit und bezogen auf alle drei Säulen genutzt werden können. Nicht zuletzt wird ja mit der Kombination der Konzepte Institutionalisierungsarbeit und feldkonfigurierende Veranstaltungen eine Vorstellung von empirischer Praxis zum Ausdruck gebracht, die davon ausgeht, dass erhaltende und verändernde Bemühungen sowie formale wie informelle Regelsetzungen bei Events gleichzeitig und durchaus konträr vorkommen. Es sind also eher die (intendierten und unintendierten) Inhalte und Effekte, mit denen letztlich eine konkrete Veranstaltung in das Schema der Tabelle 1 (Abschnitt 3.5) eingeordnet werden kann ${ }^{115}$.

Bei aller Kritik und Unausgereiftheit sehe ich Potential in dem Field-Configuring EventsAnsatz. Vor allem sind die Durchführung von Veranstaltungen an sich und das, was bei ihnen üblicherweise vonstatten geht, als Praktiken der Institutionalisierungsarbeit einschlägig. Auf die Frage „Wie und wo konkret beeinflussen denn Unternehmen ihren institutionellen Kontext?“ kann man antworten: „Unter anderem bei Veranstaltungen, an denen alle wichtigen Akteure des organisationalen Feldes beteiligt sind." Aus meinen Untersuchungen in der Halbleiterindustrie kann ich bestätigen, dass dort die Unternehmen die regelmäßigen Fachkonferenzen äußerst wichtig finden und sich intensiv auf sie vorbereiten. Sie nehmen die Ergebnisse solcher Veranstaltungen ernst und berücksichtigen sie in ihren weiteren unternehmerischen Entscheidungen: „Man hat sich ja mit seinen Technologiethemen, besonders für die Events, die einmal im Jahr stattgefunden haben wie in Pasadena, durchaus Wochen vorher mit den aktuellsten Erkenntnissen vorbereitet. ... Deswegen war der Workshop auch in dem Sinne wichtig, weil es tatsächlich auch so war, dass es

114 Lampel/Meyer (2008), S. 1028.

115 Eine detaillierte Erörterung muss hier aus Platzgründen ausbleiben und ist auch nicht Ziel des Beitrags. 
viele Firmen gegeben hat, die basierend auf diesen Ergebnissen Firmenentscheidungen getroffen haben über den weiteren finanziellen Geldfluss für entsprechende Projekte oder auch Entscheidungen, in gewisse Projekte einzusteigen" (eigenes Interview mit einem ChipHersteller, 18. März 2010).

\section{Schlussbetrachtung}

Zusammenfassend sehe ich die Forschungen zu Institutionalisierungsarbeit und feldkonfigurierenden Veranstaltungen noch in einem frühen Stadium. Insbesondere haben die Konzepte einen stark deskriptiven, allenfalls heuristischen Charakter und sind in der entsprechenden Literatur kaum zu überprüfbaren Thesen ausgearbeitet worden. Einen wichtigen nächsten Schritt in der (gemeinsamen) Entwicklung dieser Konzepte sehe ich in der differenzierten Betrachtung von Institutional Work-Formen, und zwar jeweils in Bezug zur normativen, regulativen und kulturell-kognitiven Säule in Scotts Modell, verbunden mit der Frage, welche Art von Veranstaltungen welche Form von Institutionalisierungsarbeit hinsichtlich welcher institutionellen Säule unterstützen können (siehe Abschnitte 3.5 und 4.4).

Die bisherige Unausgereiftheit der Konzepte Institutionalisierungsarbeit und feldkonfigurierende Veranstaltungen birgt zudem eine gewisse Offenheit für Einflüsse von außerhalb des Neoinstitutionalismus, was die Anschlussfähigkeit und Anwendbarkeit der Konzepte erhöht ${ }^{116}$. Die Konzepte bringen bereits jetzt für die Betriebswirtschaftslehre brauchbare Erkenntnisse und vermitteln insbesondere auch Studierenden und Praktikern einen realitätsnahen Blick auf den Umgang mit Umweltbedingungen ${ }^{117}$ :

(1) Institutionalisierungsarbeit bedeutet betriebswirtschaftlich, dass Unternehmen stets die Möglichkeit haben, mit verschiedensten Praktiken auf ihr organisationales Feld einzuwirken, statt nur innerhalb eines gegebenen Feldes optimale Entscheidungen zu treffen. Das Konzept spricht aber nicht nur gegen Steuerungspessimismus, sondern auch gegen übersteigerten Steuerungsoptimismus ${ }^{118}$, denn Unternehmen sind selbst durch ihr Feld geprägt und können nur bedingt Kreativität produzieren oder importieren und dann im Feld durchsetzen. Erfolgreich sind dann wohl die Unternehmen, die sich nicht nur anpassen, die aber auch nicht ihr Feld zerstören. Kreative Zerstörung ist in diesem Zusammenhang als Mythos zu sehen, beziehungsweise dem Zerstörungsakt als Ergebnis von Deinstitutionalisierungsarbeit müsste eine Menge konstruktiver und dann reproduktiver Institutionalisierungsarbeit folgen ${ }^{119}$.

116 Eine mögliche Richtung der Weiterentwicklung setzt am Begriff der Arbeit im Konzept der Institutionalisierungsarbeit an, um ihn ernster zu nehmen und auszudeuten - Institutions and Work, vgl. Kaghan/Lounsbury (2011). Ein entsprechender Call for Papers für ein Sonderheft der Zeitschrift Organization Studies lief Ende Februar 2011 aus.

117 Vgl. Lawrence/Suddaby/Leca (2009), S. 2, die hoffen, dass über den Begriff der Institutionalisierungsarbeit der Bogen von der neoinstitutionalistischen Theorie zur Praxis geschlagen werden kann.

118 Vgl. Sydow/Windeler (2000) zum zwischen Steuerungsoptimismus und -pessimismus liegenden Steuerungsrealismus, dort im Zusammenhang mit Unternehmensnetzwerken.

119 Vgl. Hargadon/Douglas (2001). Gerade auch eine die Actor Network Theory einbeziehende Konzeption von Institutionalisierungsarbeit wird dabei den Blick immer weniger auf einzelne Akteure (bzw. Aktanten) richten und verstärkt die Vernetztheit, Umkämpftheit, Verteiltheit und Kollektivität solcher Prozesse fokussieren, vgl. Lawrence/Suddaby (2006), S. $242 \mathrm{ff}$. 
(2) Feldkonfigurierende Veranstaltungen bedeuten betriebswirtschaftlich, dass Unternehmen stärker berücksichtigen müssen, inwieweit die in ihrem Feld üblichen Veranstaltungen wie Messen und Kongresse nicht nur Marketing im engeren Sinne ermöglichen, sondern auch ein strategisches Management der unternehmerischen Handlungsbedingungen mittels solcher Veranstaltungen ${ }^{120}$. Da es dem Unternehmen nicht egal sein kann, wohin das Feld sich entwickelt, muss es die Veranstaltungen nutzen, um die Richtung zu erkennen und möglichst zu beeinflussen. Empirisch zu überprüfen wäre sodann die Hypothese, dass der Unternehmenserfolg mit der aktiven Beteiligung an Veranstaltungen im Feld zusammenhängt. Ich vermute, dass dies stark davon abhängt, wie systematisch ein Unternehmen in diesem Bereich vorgeht und Feedbackschleifen zwischen Veranstaltungen und Tagesgeschäft installiert. Zudem ergibt sich mit der Fokussierung auf feldkonfiguriernde Veranstaltungen eine neue Perspektive auf Praktiken des Lobbying: Inwieweit wird Lobbying kollektiv über Events organisiert, die ein ganzes Feld ansprechen, oder eher doch individuell, bilateral und weniger sichtbar (zum Beispiel am Rande von Events)?

Die Neoinstitutionalistische Organisationstheorie gilt international, wie ich eingangs betont habe, als eine der einflussreichsten Theorieströmungen der letzten zehn bis zwanzig Jahre. Gerade, wenn uns diese Forschung bisher als wenig kompatibel zur deutschsprachigen Betriebswirtschaftslehre erscheinen mochte ${ }^{121}$, sind Institutionalisierungsarbeit und feldkonfigurierende Veranstaltungen erfreuliche und auch herausfordernde Konzepte, so sehr sie noch am Anfang stehen mögen und so sehr sie sich auf Probleme beziehen, die uns nicht zum ersten Mal begegnen. - Alles spricht dafür, Management auch als Institutionalisierungsarbeit zu verstehen.

\section{Literaturverzeichnis}

Aldrich, Howard E./Fiol, C. Marlene (1994), The institutional context of industry creation, in: Academy of Management Review, Vol. 19, S. 645-670.

Anand, N./Jones, Brittany C. (2008), Tournament rituals, category dynamics, and field configuration: The case of the Booker Prize, in: Journal of Management Studies, Vol. 45, S. 1036-1060.

Anand, N./Watson, Mary R. (2004), Tournament rituals in the evolution of fields: The case of the Grammy Awards, in: Academy of Management Journal, Vol. 47, S. 59-80.

Arthur, W. Brian (1994), Increasing Returns and Path Dependence in the Economy, Ann Arbor.

Barley, Stephen R./Tolbert, Pamela S. (1997), Institutionalization and structuration: Studying the links between action and institution, in: Organization Studies, Vol. 18, S. 93-117.

Battilana, Julie/D'Aunno, Thomas (2009), Institutional work and the paradox of embedded agency, in: Lawrence, Thomas B./Suddaby, Roy/Leca, Bernard (Hrsg.), Institutional Work. Actors and Agency in Institutional Studies of Organizations, Cambridge, S. 31-58.

120 Vgl. Ansätze zur „non-selling function“ von Messen bereits bei Kerin/Cron (1987), deren organisationstheoretisch informierte Ausarbeitung jedoch noch aussteht.

$121 \mathrm{Vgl}$. die ein intuitives Festhalten am entscheidungstheoretischen Paradigma suggerierenden Einwände bei Wolf (2008), S. $560 \mathrm{ff}$. 
Battilana, Julie/Leca, Bernard/Boxenbaum, Eva (2009), How actors change institutions: Towards a theory of institutional entrepreneurship, in: The Academy of Management Annals, Vol. 3, S. 65-107.

Beckert, Jens (1999), Agency, entrepreneurs, and institutional change: The role of strategic choice and institutionalized practices in organizations, in: Organization Studies, Vol. 20, S. 777-799.

Berger, Peter L./Luckmann, Thomas (1966), The Social Construction of Reality, Garden City.

Boxenbaum, Eva/Strandgaard Pedersen, Jesper (2009), Scandinavian institutionalism - a case of institutional work, in: Lawrence, Thomas B./Suddaby, Roy/Leca, Bernard (Hrsg.), Institutional Work. Actors and Agency in Institutional Studies of Organizations, Cambridge, S. 178-204.

Brown, Clair/Linden, Greg (2009), Chips and Change: How Crisis Reshapes the Semiconductor Industry, Cambridge/MA.

Child, John (1972), Organizational structure, environment and performance: The role of strategic choice, in: Sociology, Vol. 6, S. 1-22.

Cohen, Michael D./March, James G./Olsen, Johan P. (1972), A garbage can model of organizational choice, in: Administrative Science Quarterly, Vol. 17, S. 1-25.

Collier, Ruth Berins/Collier, David (1991), Shaping the Political Arena: Critical Junctures, the Labor Movement, and Regime Dynamics in Latin America, Princeton/NJ.

Dacin, M. Tina/Munir, Kamal/Tracey, Paul (2010), Formal dining at Cambridge Colleges: Linking ritual performance and institutional maintenance, in: Academy of Management Journal, Vol. 53, S. 1393-1418.

Dao, Giang/Mackay, R. Scott/Seidel, Phil (2002), NGL process and the role of International SEMATECH, in: Proceedings of SPIE, Vol. 4688, S. 29-35.

Davis, Gerald F. (2006), Mechanisms and the theory of organizations, in: Journal of Management Inquiry, Vol. 15, S. 114-118.

DiMaggio, Paul J. (1988), Interest and agency in institutional theory, in: Zucker, Lynne G. (Hrsg.), Institutional Patterns and Organizations, Cambridge/MA, S. 3-22.

DiMaggio, Paul J./Powell, Walter W. (1983), The iron cage revisited: Institutional isomorphism and collective rationality in organizational fields, in: American Sociological Review, Vol. 48, S. 147-160.

DiMaggio, Paul J./Powell, Walter W. (1991), Introduction, in: Powell, Walter W./DiMaggio, Paul J. (Hrsg.), The New Institutionalism in Organizational Analysis, Chicago, S. 1-38.

Dobusch, Leonhard/Müller-Seitz, Gordon (2011), Serial singularities: Developing a network organization by organizing events, angenommen zur Veröffentlichung in: Schmalenbach Business Review.

Dorado, Silvia (2005), Institutional entrepreneurship, partaking, and convening, in: Organization Studies, Vol. 26, S. 385-414.

Douglas, Mary (1970), Natural Symbols: Explorations in Cosmology, Harmondsworth.

Emirbayer, Mustafa/Mische, Ann (1998), What is agency?, in: American Journal of Sociology, Vol. 103, S. 962-1023.

Garud, Raghu (2008), Conferences as venues for the configuration of emerging organizational fields: The case of cochlear implants, in: Journal of Management Studies, Vol. 45, S. 1061-1088.

Garud, Raghu/Hardy, Cynthia/Maguire, Steve P. (2007), Institutional entrepreneurship as embedded agency: An introduction to the special issue, in: Organization Studies, Vol. 28, S. 957-969.

Garud, Raghu/Jain, Sanjay/Kumaraswamy, Arun (2002), Institutional entrepreneurship in the sponsorship of common technological standards: The case of Sun Microsystems and Java, in: Academy of Management Journal, Vol. 45, S. 196-214.

Garud, Raghu/Karnøe, Peter (2003), Bricolage versus breakthrough: Distributed and embedded agency in technology entrepreneurship, in: Research Policy, Vol. 32, S. 277-300.

Giddens, Anthony (1979), Central Problems in Social Theory: Action, Structure and Contradiction in Social Analysis, London.

Giddens, Anthony (1984), The Constitution of Society, Berkeley. 


\section{G. MÖLLERING}

Goffman, Erving (1959), The Presentation of Self in Everyday Life, London.

Goffman, Erving (1967), Interaction Ritual: Essays on Face-to-Face Behavior, Garden City.

Granovetter, Mark (1985), Economic action and social structure: The problem of embeddedness, in: American Journal of Sociology, Vol. 91, S. 481-510.

Greenwood, Royston/Oliver, Christine/Sahlin, Kerstin/Suddaby, Roy (2008), Introduction, in: Greenwood, Royston/Oliver, Christine/Sahlin, Kerstin/Suddaby, Roy (Hrsg.), The SAGE Handbook of Organizational Institutionalism, London, S. 1-46.

Greenwood, Royston/Suddaby, Roy (2006), Institutional entrepreneurship in mature fields: The big five accounting firms, in: Academy of Management Journal, Vol. 49, S. 27-48.

Hardy, Cynthia/Maguire, Steve (2008), Institutional entrepreneurship, in: Greenwood, Royston/Oliver, Christine/Sahlin, Kerstin/Suddaby, Roy (Hrsg.), The SAGE Handbook of Organizational Institutionalism, London, S. 198-217.

Hardy, Cynthia/Maguire, Steve (2010), Discourse, field-configuring events, and change in organizations and institutional fields: Narratives of DDT and the Stockholm Convention, in: Academy of Management Journal, Vol. 53, S. $1365-1392$.

Hargadon, Andrew B./Douglas, Yellowlees (2001), When innovations meet institutions: Edison and the design of the electric light, in: Administrative Science Quarterly, Vol. 46, S. 476-501.

Hoffman, Andrew J. (1999), Institutional evolution and change: Environmentalism and the U.S. chemical industry, in: Academy of Management Journal, Vol. 42, S. 351-371.

Holm, Petter (1995), The dynamics of institutionalization: Transformation processes in Norwegian fisheries, in: Administrative Science Quarterly, Vol. 40, S. 398-422.

Hwang, Hokyu/Colyvas, Jeannette A. (2011), Problematizing actors and institutions in institutional work, in: Journal of Management Inquiry, Vol. 20, S. 62-66.

Jepperson, Ronald L. (1991), Institutions, institutional effects, and institutionalism, in: Powell, Walter W. /DiMaggio, Paul J. (Hrsg.), The New Institutionalism in Organizational Analysis, Chicago, S. 143-163.

Kaghan, William/Lounsbury, Michael (2011), Institutions and work, in: Journal of Management Inquiry, Vol. 20, S. 73-81.

Kerin, Roger A./Cron, William L. (1987), Assessing trade show functions and performance: An exploratory study, in: Journal of Marketing, Vol. 51, S. 87-94.

Kraatz, Matthew S. (2011), Two cheers for institutional work, in: Journal of Management Inquiry, Vol. 20, S. 59-61.

Lampel, Joseph (2011), Afterword: Converting values into other values: Fairs and Festivals as resource valuation and trading events, in: Moeran, Brian/Strandgaard Pedersen, Jesper (Hrsg.), Negotiating Values in the Creative Industries: Fairs, Festivals and Competitive Events, Cambridge, S. 334-347.

Lampel, Joseph/Meyer, Alan D. (2008), Field-configuring events as structuring mechanisms: How conferences, ceremonies, and trade shows constitute new technologies, industries, and markets, in: Journal of Management Studies, Vol. 45, S. 1025-1035.

Lawrence, Thomas B. (1999), Institutional strategy, in: Journal of Management, Vol. 25, S. 161-188.

Lawrence, Thomas B./Suddaby, Roy (2006), Institutions and institutional work, in: Clegg, Stewart R./Hardy, Cynthial Lawrence, Thomas B./Nord, Walter R. (Hrsg.), The Sage Handbook of Organization Studies, London, S. $215-254$.

Lawrence, Thomas B./Suddaby, Roy/Leca, Bernard (2009), Introduction: Theorizing and studying institutional work, in: Lawrence, Thomas B./Suddaby, Roy/Leca, Bernard (Hrsg.), Institutional Work. Actors and Agency in Institutional Studies of Organizations, Cambridge, S. 1-27.

Lawrence, Thomas B./Suddaby, Roy/Leca, Bernard (2011), Institutional work: Refocusing institutional studies of organization, in: Journal of Management Inquiry, Vol. 20, S. 52-58.

Lawrence, Thomas B./Winn, Monika I./Jennings, P. Devereaux (2001), The temporal dynamics of institutionalization, in: Academy of Management Review, Vol. 26, S. 624-644. 
Löwer, Ulrich M. (2005), Interorganisational Standards: Managing Web Services Specifications for Flexible Supply Chains, Heidelberg/New York.

Meyer, Alan D./Gaba, Vibha/Colwell, Kenneth A. (2005), Organizing far from equilibrium: Nonlinear change in organizational fields, in: Organization Science, Vol. 16, S. 456-473.

Meyer, John W.IJepperson, Ronald L. (2000), The 'actors' of modern society: The cultural construction of social agency, in: Sociological Theory, Vol. 18, S. 100-120.

Meyer, John W./Rowan, Brian (1977), Institutionalized organizations: Formal structure as myth and ceremony, in: American Journal of Sociology, Vol. 83, S. 340-363.

Möllering, Guido (2010), Collective Market-Making Efforts at an Engineering Conference, MPIfG Discussion Paper 10/2, Köln.

Moeran, Brian/Strandgaard Pedersen, Jesper (Hrsg.) (2011), Negotiating Values in the Creative Industries: Fairs, Festivals and Competitive Events, Cambridge.

Moore, Gordon E. (1965), Cramming more components onto integrated circuits, in: Electronics, Vol. 38, S. 114-117.

North, Douglass C. (1990), Institutions, Institutional Change and Economic Performance, Cambridge.

Oliver, Christine (1991), Strategic responses to institutional processes, in: Academy of Management Review, Vol. 16, S. 145-179.

Oliver, Christine (1992), The antecedents of deinstitutionalization, in: Organization Studies, Vol. 13, S. 563-588.

Ortmann, Günther/Zimmer, Marco (1998), Strategisches Management, Recht und Politik, in: Die Betriebswirtschaft, 58. Jg., S. 747-769.

Phillips, Nelson (2011), Editor's introduction, in: Journal of Management Inquiry, Vol. 20, S. 51.

Rojas, Fabio (2010), Power through institutional work: Acquiring academic authority in the 1968 Third World Strike, in: Academy of Management Journal, Vol. 53, S. 1263-1280.

Schiller-Merkens, Simone (2008), Institutioneller Wandel und Organisationen: Grundzüge einer strukturationstheoretischen Konzeption, Wiesbaden.

Scott, W. Richard (1994), Conceptualizing organizational fields: Linking organizations and societal systems, in: Derlien, Hans-Ulrich/Gerhardt, Uta/Scharpf, Fritz W. (Hrsg.), Systemrationalität und Partialinteresse, Baden-Baden, S. 203-221.

Scott, W. Richard (2008), Institutions and Organizations, 3. Aufl., Thousand Oaks/CA.

Selznick, Philip (1996), Institutionalism "Old" and "New", in: Administrative Science Quarterly, Vol. 41, S. 270-277.

Senge, Konstanze (2011), Das Neue am Neo-Institutionalismus: Der Neo-Institutionalismus im Kontext der Organisationswissenschaft, Wiesbaden.

Seo, Myeong-Gu/Creed, W. E. Douglas (2002), Institutional contradictions, praxis, and institutional change: A dialectical perspective, in: Academy of Management Review, Vol. 27, S. 222-247.

Suddaby, Roy (2010), Challenges for institutional theory, in: Journal of Management Inquiry, Vol. 19, S. 14-20.

Suddaby, Roy/Elsbach, Kimberly D./Greenwood, Royston/Meyer, John W./Zilber, Tammar B. (2010), Organizations and their institutional environments - Bringing meaning, values, and culture back in: Introduction to the Special Research Forum, in: Academy of Management Journal, Vol. 53, S. 1234-1240.

Suchman, Mark (1995), Managing legitimacy: Strategic and institutional approaches, in: Academy of Management Review, Vol. 20, S. 571-610.

Sydow, Jörg/Schreyögg, Georg/Koch, Jochen (2009), Organizational path dependence: Opening the black box, in: Academy of Management Review, Vol. 34, S. 689-709.

Sydow, Jörg/Windeler, Arnold (2000), Steuerung von und in Netzwerken - Perspektiven, Konzepte, vor allem aber offene Fragen, in: Sydow, Jörg/Windeler, Arnold (Hrsg.), Steuerung von Netzwerken: Konzepte und Praktiken, Wiesbaden, S. 1-24.

Tolbert, Pamela S./Zucker, Lynne G. (1996), The institutionalization of institutional theory, in: Clegg, Stewart R./ Hardy, Cynthia/Nord, Walter R. (Hrsg.), Handbook of Organization Studies, London, S. 175-190. 


\section{G. MÖLLERING}

Walgenbach, Peter (2002), Neoinstitutionalistische Organisationstheorie - State of the Art und Entwicklungslinien, in: Schreyögg, Georg/Conrad, Peter (Hrsg.), Managementforschung 12: Theorien des Managements, Wiesbaden, S. 155-202.

Walgenbach, Peter (2006), Neoinstitutionalistische Ansätze in der Organisationstheorie, in: Kieser, Alfred/Ebers, Mark (Hrsg.), Organisationstheorien, Stuttgart, S. 353-401.

Walgenbach, Peter/Meyer, Renate E. (2008a), Institutional entrepreneurship and the structuring of organizations and markets, in: Ebner, Alexander/Beck, Nikolaus (Hrsg.), The Institutions of the Market: Organizations, Social Systems, and Governance, Oxford, S. 180-201.

Walgenbach, Peter/Meyer, Renate E. (2008b), Neoinstitutionalistische Organisationstheorie, Stuttgart.

Warren, Ronald L. (1967), The interorganizational field as a focus for investigation, in: Administrative Science Quarterly, Vol. 12, S. 396-419.

Weber, Max (1978), Die protestantische Ethik und der Geist des Kapitalismus, Tübingen.

Wijen, Frank/Ansari, Shahzad (2007), Overcoming inaction through collective institutional entrepreneurship: Insights from regime theory, in: Organization Studies, Vol. 28, S. 1079-1100.

Willmott, Hugh (2011), "Institutional Work" for what? Problems and prospects of institutional theory, in: Journal of Management Inquiry, Vol. 20, S. 67-72.

Wöhe, Günter/Döring, Ulrich (2010), Einführung in die Allgemeine Betriebswirtschaftslehre, 24. Aufl., München.

Wolf, Joachim (2008), Organisation, Management, Unternehmensführung: Theorien, Praxisbeispiele und Kritik, 3. Aufl., Wiesbaden.

Wooten, Melissa/Hoffman, Andrew J. (2008), Organizational fields: Past, present and future, in: Greenwood, Royston/ Oliver, Christine/Sahlin, Kerstin/Suddaby, Roy (Hrsg.), The SAGE Handbook of Organizational Institutionalism, London, S. 130-147.

Zietsma, Charlene/Lawrence, Thomas B. (2010), Institutional work in the transformation of an organizational field: The interplay of boundary work and practice work, in: Administrative Science Quarterly, Vol. 55, S. 189-221.

Zietsma, Charlene/McKnight, Brent (2009), Building the iron cage: Institutional creation work in the context of competing proto-institutions, in: Lawrence, Thomas B./Suddaby, Roy/Leca, Bernard (Hrsg.), Institutional Work. Actors and Agency in Institutional Studies of Organizations, Cambridge, S. 143-177.

Zilber, Tammar B. (2007), Stories and the discursive dynamics of institutional entrepreneurship: The case of Israeli high-tech after the bubble, in: Organization Studies, Vol. 28, S. 1035-1054.

Zucker, Lynne G. (1977), The role of institutionalization in cultural persistence, in: American Sociological Review, Vol. 42, S. 726-743.

Zucker, Lynne G. (1987), Institutional theories of organization, in: Annual Review of Sociology, Vol. 13, S. 443-464.

Zukin, Sharon/DiMaggio, Paul J. (1990), Introduction, in: DiMaggio, Paul J./Zukin, Sharon (Hrsg.), Structures of Capital: The Social Organization of the Economy, Cambridge, S. 1-36.

\section{Summary}

The paper discusses two new concepts in Neoinstitutional Organization Theory: Institutional Work and Field-Configuring Events. This stream of institutional theorizing moves toward a practice-theoretical turn. It grants firms more opportunities for influencing their institutional environments than the prior neoinstitutional determinism, but without adopting methodological individualism. The paper analyzes critically the sources and background of this theoretical development and reaches the moderately optimistic conclusion that Business Administration will benefit theoretically and methodologically from understanding management as Institutional Work. 